\title{
Modelling trip distribution with fuzzy and genetic fuzzy systems
}

\author{
Mert Kompil ${ }^{\mathrm{a}, \mathrm{b} *}$ and H. Murat Celik ${ }^{\mathrm{b}}$ \\ ${ }^{a}$ European Commission, Joint Research Centre (JRC), Institute for Prospective Technological \\ Studies (IPTS), Cl Inca Garcilaso 3, 41092, Seville, Spain; ${ }^{b}$ Department of City and Regional \\ Planning, Izmir Institute of Technology, 35430, Izmir, Turkey
}

(Received 23 January 2012; accepted 12 October 2012)

\begin{abstract}
This paper explores the potential capabilities of fuzzy and genetic fuzzy system approaches in urban trip distribution modelling with some new features. First, a simple fuzzy rule-based system (FRBS) and a novel genetic fuzzy rule-based system [GFRBS: a fuzzy system improved by a knowledge base learning process with genetic algorithms (GAs)] are designed to model intra-city passenger flows for Istanbul. Subsequently, their accuracy, applicability and generalizability characteristics are evaluated against the well-known gravity- and neural network (NN)-based trip distribution models. The overall results show that: traditional doubly constrained gravity models are still simple and efficient; NNs may not show expected performance when they are forced to satisfy trip constraints; simply-designed FRBSs, learning from observations and expertise, are both efficient and interpretable even if the data are large and noisy; and use of GAs in fuzzy rule-based learning considerably increases modelling performance, although it brings additional computation cost.
\end{abstract}

Keywords: trip distribution; spatial interaction models; fuzzy logic; fuzzy rulebased systems; genetic fuzzy systems; genetic algorithms; neural networks

\section{Introduction}

In recent decades, many techniques have been proposed for each step of travel demand analysis. Among them, trip distribution has probably been the most attractive field of demand analysis, especially given the widespread use of gravity models. Transportation researchers and practitioners have recently become more interested in exploring the capability of applying soft computing (computationally intelligent) techniques to real transportation problems. Research in more effective and predictive methodologies in spatial interaction and trip distribution modelling has also led to some pioneering studies in this area. Many scholars have proposed new modelling procedures to forecast aggregate interactions using neural networks (NNs), fuzzy logic (FL) and genetic algorithms (GAs). The initial experiences with these techniques have been encouraging, and the overall results suggest that NNs, FL and GAs can be used successfully in spatial interactions models.

From our point of view, use of fuzzy set theory and FL are very promising in modelling spatial interactions for several reasons: (1) these methods are simple, flexible and equation-free; (2) they provide an opportunity to incorporate expert knowledge into the modelling procedure, a process that may increase the interpret-

*Corresponding author. Email: Mert.kompil@ec.europa.eu 
ability of the analysed system; and (3) they are capable of increasing the accuracy of the estimations when hybridized with GAs or NNs. The pioneering studies of Kalic and Teodorovic $(1996,2003)$ showed the prospective use of fuzzy and genetic fuzzy systems (GFSs) in trip distribution modelling. They designed a fuzzy rule-based system (FRBS) and a genetic fuzzy rule-based system (GFRBS) in their consecutive studies to estimate air passenger flows from origin outflows and destination inflows. However, in comparison with the approaches centred on gravity models and NNs, the full potential of FRBSs and GFRBSs has still not been demonstrated in trip distribution modelling. Their efficiency, accuracy, applicability and interpretability are still under investigation. In particular, the applicability of GFRBSs to the estimation of intra-city passenger flows has not previously been investigated. They offer high-quality predictions, but their computational challenges with an additional friction variable are not known.

With this background, this paper attempts to set out an FRBS and a GFRBS for modelling intra-city passenger flows in Istanbul. Our primary interest was to contribute to the knowledge and literature on using such models for urban trip distribution modelling. Another objective was to compare and evaluate the accuracy, applicability and generalizability of such models to that of well-known trip distribution models in a complex real-world case. For this purpose, a doubly constrained gravity model (DCGM) and a multilayer feed-forward NN-based trip distribution model were established as the benchmarks, against which model performances were evaluated empirically using the 2006 Istanbul Travel Survey data.

In the following two sections, we provide a brief review of previous studies in trip distribution modelling. Description of the study area and data, fundamentals of the FRBSs and GFRBSs, the methodology for proposed and benchmark models and performance criteria are then introduced in the empirical analysis section. We conclude with a discussion of the significance of the empirical findings.

\section{Conventional solution to a trip distribution problem}

In the simplest terms, any trip distribution model estimates the number of trips between given origins and destinations. From the early 1950s, modellers have used several different formulations and variables to deal with this task. The use of initial growth factor models was followed by the inception of gravity models by the mid1950 s and then by many other aggregate or disaggregate models (including intervening opportunity models, random utility models and activity-based models). All models have a theoretical basis in physics, statistics, economics and behavioural sciences. The interested reader is referred to Ortuzar and Willumsen (2001), Black (2003), Kanafani (1983) and Oppeneim (1995) for an in-depth coverage of these theoretical models.

With its well-known theoretical basis and various application procedures, the gravity type of spatial interaction model has been the most commonly used trip distribution model. Gravity models simply assume [analogously to Newton's Law of Universal Gravitation (1686)] that the interaction between any two zones is directly proportional to their magnitudes and inversely proportional to the distance between them. Over the years, this basic assumption has improved with new theoretical insights, variables and functional forms. In particular, Wilson's $(1967,1970)$ doubly constrained model has found wide applicability among practitioners in modelling 
aggregate distributions. In a classical manner, the expression of a DCGM for the trip distribution problem can be stated as follows:

$$
\begin{aligned}
\mathrm{T}_{\mathrm{ij}} & =a_{i} b_{j} P_{i} A_{j} f\left(C_{i j}\right) \\
\mathrm{a}_{\mathrm{i}} & =1 / \sum_{j} b_{j} A_{j} f\left(C_{i j}\right) \\
\mathrm{b}_{\mathrm{j}} & =1 / \sum_{i} a_{i} P_{i} f\left(C_{i j}\right)
\end{aligned}
$$

where $T_{i j}$ is the number of trips from zone $i$ to zone $j, P_{i}$ is the total number of trips produced in zone $i, A_{j}$ is the total number of trips attracted to zone $j, f\left(C_{i j}\right)$ is the friction factor related to some measure of spatial separation between zone $i$ and zone $j$ and finally $a_{i}$ and $b_{j}$ are the balancing factors that ensures origin $\left(\sum_{j} T_{i j}=P_{i}\right)$ and destination $\left(\sum_{i} T_{i j}=A_{j}\right)$ constraints are satisfied.

The spatial separation of zones is usually included in the model as a cost of physical distance or travel time. Once the friction parameter(s) have been calibrated for the base year trip matrix, the future pattern of trips can be easily simulated. The well-known friction functions are: (1) exponential-cost function, $f\left(C_{i j}\right)=e^{-\beta\left(C_{i j}\right)}$, (2) power-cost function, $f\left(C_{i j}\right)=C_{i j}^{-\beta}$ and (3) gamma or combined-cost function, $f\left(C_{i j}\right)=e^{-\beta\left(C_{i j}\right)} C_{i j}^{-\beta}$.

There is a considerable amount of literature on gravity and spatial interaction models that is outside the scope of this study. Fotheringham and O'Kelly (1989), Sen and Smith (1995) and Roy (2004) provide excellent reviews.

\section{Modelling trip distribution with soft computing techniques: a review of past studies}

Many researchers have explored the feasibility of applying soft computing techniques to real transportation problems. Lotfi Zadeh, the founder of fuzzy set theory, describes soft computing as '.. a collection of methodologies, which in one form or another reflect the guiding principle of soft computing: exploit the tolerance for imprecision, uncertainty, and partial truth to achieve tractability, robustness, and low solution cost' (Pedrycz, 1996). In parallel with other engineering sciences, NNs, FL and GAs have been the most featured soft computing techniques used in transportation research. A number of studies have shown the applicability of these techniques to disciplines such as traffic control and management, travel demand analysis and transport logistics. Some of the more important traffic and transportation applications of soft computing techniques have been cited in Teodorovic and Vukadinovic (1998), Avineri (2005) and Transportation Research Board (2007). Many scholars have also proposed new modelling procedures to forecast trip distributions with soft computing. The following two sections briefly review their work.

\section{Modelling trip distribution with NNs}

$\mathrm{NNs}$ are computational models of the human brain. Artificial neurons are interconnected by edges constituting a layered network; the network receives input, performs some internal process such as activations of the neurons, and produces output (Munakata, 2008). Multilayer $\mathrm{NNs}$ are, at the same time, universal approximators (Hornik, Stinchcombe and White, 1989; Hornik, 1991). Once the 
connection weights of the neurons are adjusted, in other words 'trained', they are able to approximate any nonlinear function accurately (Sadek, 2007).

The application potentials of NNs have been shown in many scientific disciplines as well as in transport geography and modelling [see Dougherty (1995) and Mussone (1999) for a review of NNs in transport geography and traffic engineering, and valuable works by Munakata (2008) and Haykin (1999) for a detailed theoretical framework of NNs].

Several recent studies have also proposed use of NNs to model spatial interactions and trip distribution. Openshaw (1993) presented the potential use of NNs in spatial interaction modelling and Fischer and Gopal (1994) showed the applicability and predictive accuracy of NNs in modelling the distribution of interregional telecommunication flows. Many others have followed these pioneering works in trip distribution modelling: Black (1995) and Celik (2004) modelled commodity flows and Mozolin, Thill, and Lynn Usery (2000), Tillema, van Zuilekom, and van Maarseveen (2006) and Tapkin and Aky1lmaz (2009) modelled intercity passenger flows with NNs.

Nearly all scholars compared the NNs predictive performance with conventional spatial interaction models, which are generally unconstrained. In many cases, NNs outperformed the conventional models, leading to the conclusion that NNs may perform well enough to estimate spatial interaction flows in general. The only differentiating conclusion was presented by Mozolin, Thill, and Lynn Usery (2000) and Celik (2004). They concluded that NNs may perform better than conventional models for a base year matrix, but they fail to outperform conventional models for forecasting purposes.

\section{Modelling trip distribution with fuzzy and GFSs}

Fuzzy set theory and FL were first introduced by Lotfi Zadeh in 1965 and 1973 as mathematical tools for dealing with uncertainty, imprecision and subjectivity, stated in linguistic terms. Since then, a number of studies have been performed and a number of practical engineering applications have been established using the concept of fuzziness. FL has proved to be a good tool for a wide range of application areas such as system/process control, pattern recognition, classification, machine learning, decision-making and approximate reasoning. It has also been proven to be very useful in nonlinear input-output mapping since Wang and Mendel (1992) and Kosko (1994) showed in their works that fuzzy systems can be treated as universal approximators.

FRBSs are one of the most important application areas of fuzzy set theory. As an extension of classical rule-based systems, an FRBS uses fuzzy sets and FL to represent and connect knowledge which is usually linguistic in nature. They have also been applied in many traffic and transportation studies. Traffic control and management, accident analysis and prevention, selection of transportation investments, and modelling any individual step of the many steps comprising travel demand analysis are widely known application areas of FRBSs (Teodorović, 1994, 1999).

Apart from these applications, learning fuzzy rules and tuning fuzzy MFs are the two key components for an FRBS. GAs have proven suitable for solving both combinatory optimisation and parameter optimisation problems. Employing GAs to 
construct a fuzzy system which learns from examples can greatly enhance the control performance of a fuzzy system (Chiou and Lan, 2005). This line of research has spurred the broad use of fuzzy systems improved by a GA learning process: GFS and in particular GFRBSs (see Cordón et al., 2004; Ishibuchi, 2007; Herrera, 2008).

With respect to trip distribution modelling, it is possible to examine several important studies that achieved significant results using FRBSs or GFRBSs. A pioneering FL approach to trip distribution modelling was introduced by Kalic and Teodorovic (1996). They estimated air passenger flows among selected major industrial cities and tourist resorts using known productions and attractions as inputs. Their proposed FRBS produced better results than those of conventional non-fuzzy methods. In another, Kalić and Teodorovic (2003) improved their work achieving better results with a GFRBS design. Finally, Shafahi, Nourbakhsh, and Seyedabrishami (2008) proposed an FRBS to predict the number of discretionary trips in Tehran and showed its capability in predicting intra-city passenger flows. They used travel time as an additional input and gained better results against an unconstrained gravity model.

According to these studies, FRBSs can be used to solve trip distribution problems efficiently and, together with the use of GAs, it is possible to achieve better model performances. However, the performance of FRBSs against a DCGM and a NNbased trip distribution model has not yet been investigated. Moreover, the GFRBS has still not been adapted for the prediction of intra-city passenger flows, which adds computational burden and complexity with an additional friction variable and additional fuzzy rules. The present study tries to make up for these shortages with an empirical analysis.

\section{Empirical analysis}

\section{Description of the study area and data}

The Istanbul metropolitan area was selected for the case study. It is a very complex and challenging city region on which to test a trip distribution model. Its transportation system consists of a high number of interaction links, nodes and bridge crossings. Moreover, the production-attraction and friction matrices of the metropolitan area were measured recently, in a large household survey.

Istanbul is located in northwest Turkey connecting the Marmara and Black Seas and separating two continents: Asia and Europe. It has a population of nearly 13.8 million, or $17.8 \%$ of Turkey's total population (TurkStat, 2010). An estimated 21 million daily trips occur in the Istanbul metropolitan area. Fifty per cent of these trips are by foot, $14 \%$ by private cars and $36 \%$ by public transit modes. Additionally, 1.3 million daily trips are continent crossings, 1 million on bridges and the remaining 300,000 with ferries (Istanbul Metropolitan Municipality Transportation Planning Department, 2008).

The data used in this study come from a Household Travel Survey conducted by the Transportation Department of the Istanbul Metropolitan Municipality in 2006. The survey was established for 451 Traffic Analysis Zones (TAZs) covering the entire metropolitan area of Istanbul and including 90,000 households (3\% sampling rate). In the survey, which had an $80 \%$ response rate, approximately 264,000 people in 
72,000 households were surveyed and a total of 356,000 trips were recorded between the 451 origin-destination pairs.

The observed trips (including both pedestrian and motorised trips) between a possible 203,401 distinct interaction points were categorized by trip purpose. Approximately 127,000 of these trips were for home-based-work (HBW), 94,000 trips for home-based-school (HBS), 115,000 trips for home-based-other (HBO) and 20,000 trips for non-home-based (NHB) trips.

Use of HBW trips was found sufficient for empirical analysis. The use of the production-attraction (P-A) form of the HBW trip matrix was preferred for the modelling procedure. Figure 1 shows the spatial distribution of zonal production and attraction totals of $\mathrm{HBW}$ trips in the Istanbul metropolitan area. The trip matrix includes both within and between TAZ interactions. Additionally, assigned travel
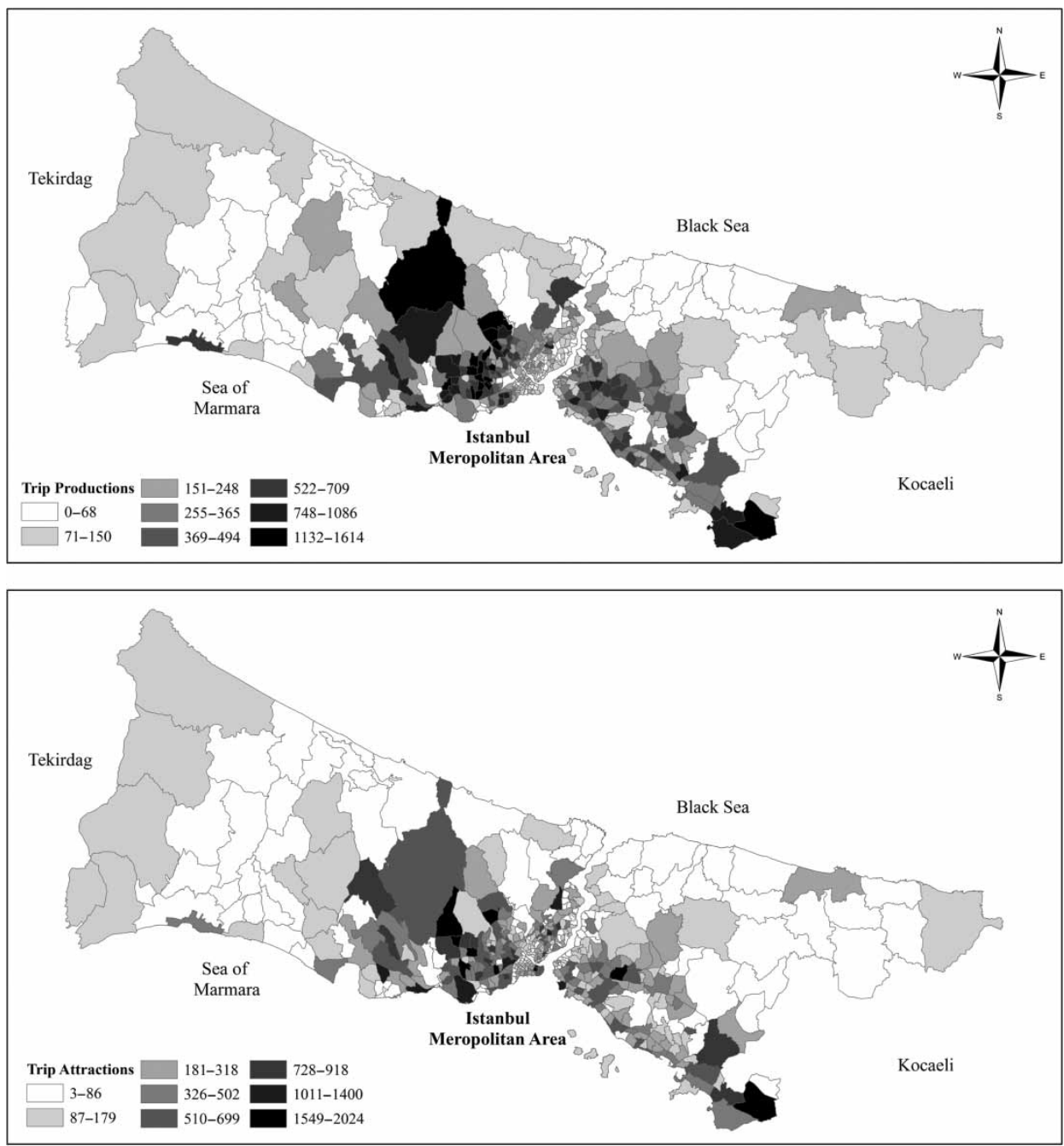

Figure 1. Zonal production and attraction totals of HBW trips in Istanbul Metropolitan Area. 
times are chosen due to typical problems with travel time self-reported in the survey. In summary, the modelling data are constituted from two 451 by 451 matrices: a P-A trip matrix and a travel time matrix.

For calibration and modelling purposes, the whole HBW matrix is divided into two equally representative rectangle matrices: a training matrix, and a testing matrix. The training matrix includes trips from all TAZs to odd numbered TAZs (a 451 by 226 matrix); the testing matrix includes trips from all TAZs to even numbered TAZs (a 451 by 225 matrix). The representativeness of two datasets is further tested with a two-sample paired $t$-test. No statistically significant difference was found between training, testing and whole datasets with respect to the trip length distributions (TLDs). Observed TLDs of all three datasets are shown in Figure 2.

\section{Modelling trip distribution with a FRBS}

\section{Main components of FRBSs}

FRBSs - also known as fuzzy inference, fuzzy control or fuzzy expert systems - are most useful in modelling complex systems that can be observed by humans. The most common way to represent human knowledge is to form it into natural language expressions: IF antecedent, THEN consequent. This expression is commonly referred as an IF-THEN rule-based system. It enables use of linguistic variables as antecedents and consequents, and logical connectives and, or, and not as well. Using basic properties of fuzzy sets and fuzzy relations, any compound rule structure can be decomposed to a number of simple canonical rules enabling approximation of any non-linear system outputs (Ross, 2004).

The most commonly used FRBSs can be distinguished into two main configurations: (1) Mamdani-type FRBS, proposed by Mamdani (1974) and Mamdani and Assilian (1975) and (2) Sugeno-type FRBS, introduced by Takagi and Sugeno (1985) and Sugeno and Kang (1988). They are all similar in their antecedents and rule-based

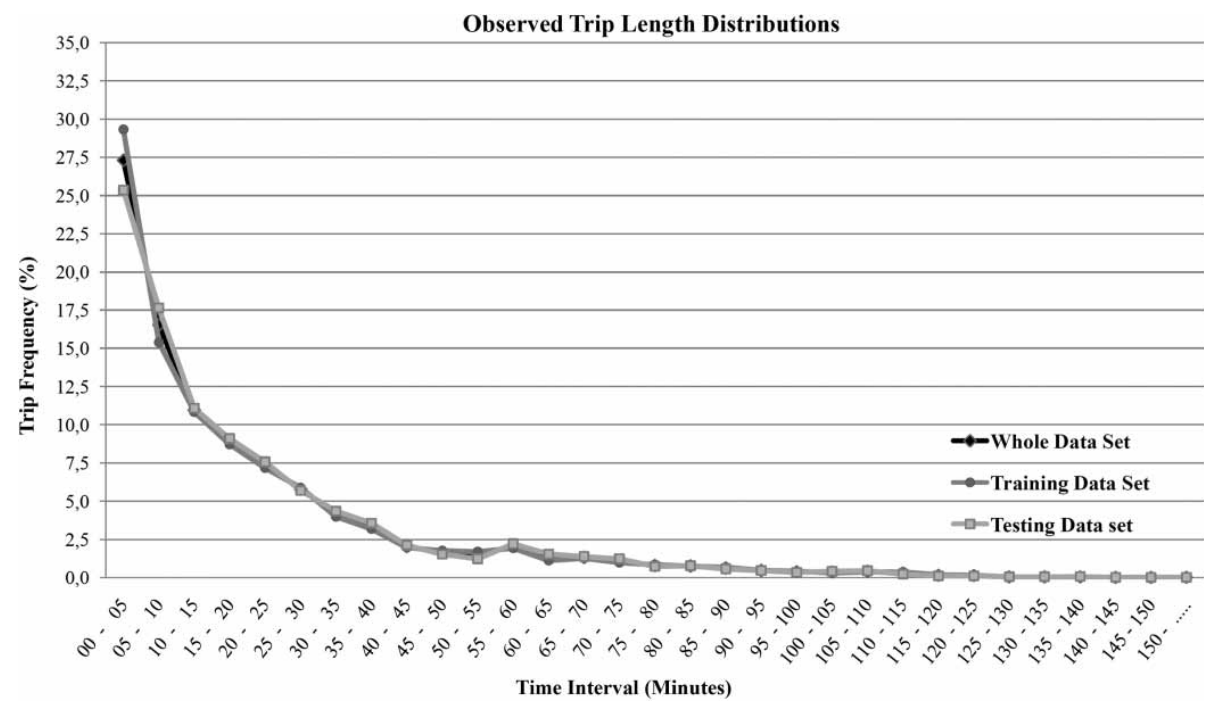

Figure 2. Observed TLDs of datasets. 
structures, and their ability to process human like reasoning with linguistic variables. However, the system outputs they produce are different. The output of a Mamdanitype FRBS is a fuzzy set, whereas the output of a Sugeno-type FRBS is generally either a linear function of its inputs or some constants. An approximate fuzzy rule can be formed as follows for these two distinct FRBS systems:

$$
\begin{aligned}
& \text { Mamdani - type FRBS : IF } \mathrm{X}_{1} \text { is } \mathrm{A}_{1} \text { and } \mathrm{X}_{2} \text { is } \mathrm{A}_{2} \text { and } \ldots \mathrm{X}_{\mathrm{i}} \text { is } \mathrm{A}_{\mathrm{i}} \text {, } \\
& \text { THEN } Y \text { is } B \\
& \text { Sugeno - type FRBS : IF } \mathrm{X}_{1} \text { is } \mathrm{A}_{1} \text { and } \mathrm{X}_{2} \text { is } \mathrm{A}_{2} \text { and } \ldots \mathrm{X}_{\mathrm{i}} \text { is } \mathrm{A}_{\mathrm{i}} \text {, } \\
& \text { THEN } Y=a_{0}+a_{1} X_{1}+a_{2} X_{2}+\ldots a_{i} X_{i}
\end{aligned}
$$

The Mamdani-type FRBS used in this study is the most common FRBS in practice and in the literature. It generally deals with mapping crisp inputs into crisp outputs and enables the use of linguistic variables and expert knowledge. This knowledge can easily be combined with automatically generated rules from data sets that describe the relationship between system input and output. Either Mamdani-type or Sugenotype, an FRBS generally consists of four main components or steps: fuzzification, knowledge base, inference and defuzzification as indicated by grey boxes in Figure 3. An explanation for these steps can be seen in Ross (2004) and Cordon et al. (2001).

\section{The proposed FRBS design}

FRBSs are useful in two general contexts: (1) in situations involving uncertainty, imprecision and partial truth and (2) in situations where investigators are mapping any inputs into desired outputs even if there is no uncertainty and imprecision present. In this study, we propose a Mamdani-type FRBS designed in the latter context to solve urban trip distribution problem.

The main logic behind the proposed FRBS design and its variable structure are the same as with the classical gravity model including three inputs and one output. Considering an origin-destination zone pair, if zonal trip productions and trip

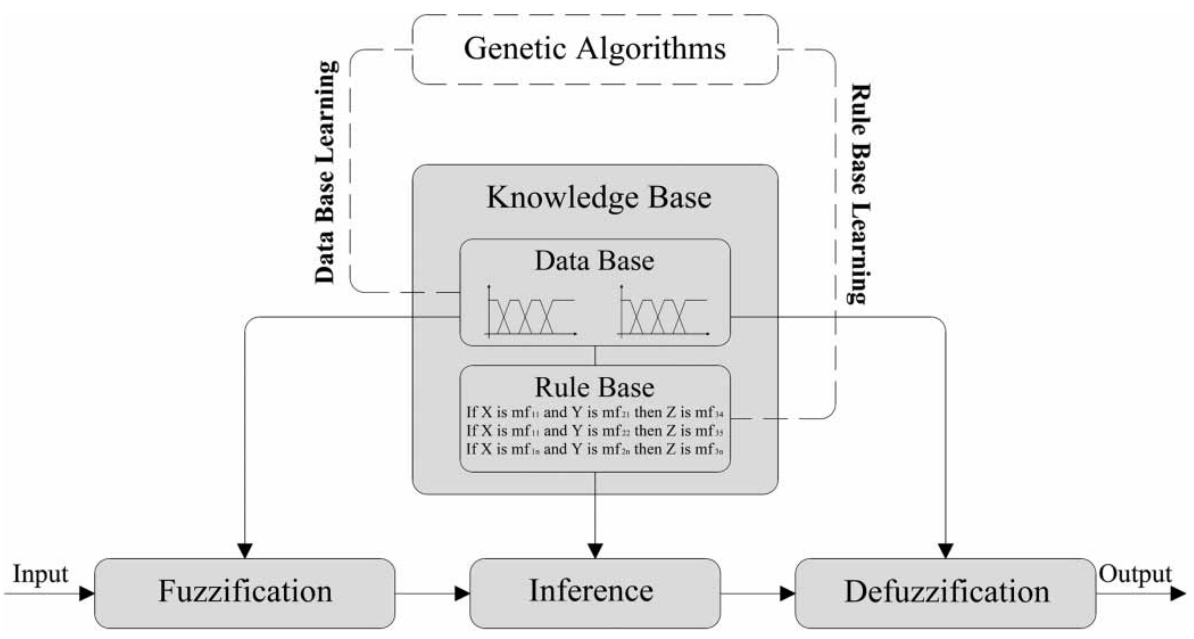

Figure 3. Main components of FRBSs and GFRBs. 
attractions are known along with the corresponding friction factor, the number of interactions/trips between this zone pair can be estimated using a FRBS as a universal approximator. Its main structure can be established starting from the simple verbal statements as follows:

(1) IF total trip production of the origin is LOW, AND total trip attraction of the destination is LOW, AND friction factor between corresponding origin and destination is HIGH, THEN the interactions/trips between origin and destination is LOW;

(2) IF total trip production of the origin is HIGH, AND total trip attraction of the destination is $\mathrm{HIGH}$, AND friction factor between corresponding origin and destination is LOW, THEN the interactions/trips between origin and destination is HIGH.

In order to construct a FRBS, these verbal statements are to be decomposed into a set of overlapping fuzzy sets connected to If-Then rules with logical operators. There are several ways to establish fuzzy partitions and a fuzzy rule base.

The present study deals with designing a simple and effective FRBS that is mindful of the accuracy-interpretability trade-off. Therefore, a heuristic design of fuzzy sets with few partitions is preferred for simplicity. Then, a widely-used WangMendel method (1992), also known as the one-pass method, is implied as the fuzzy rule induction procedure. The following five steps describe the construction and training of the proposed FRBS design:

Divide input-output spaces into overlapping regions:

The input-output pairs of trip distribution problem can be stated as,

$$
\left(P_{1}, A_{1}, F_{1} ; T_{1}\right),\left(P_{2}, A_{2}, F_{2} ; T_{2}\right), \ldots,\left(P_{i}, A_{i}, F_{i} ; T_{i}\right)
$$

where $\mathrm{P}, \mathrm{A}$ and $\mathrm{F}$ (production, attraction and friction) represent input and $\mathrm{T}$ (trips) represent output variables. Each variable has a domain interval which lies between minimum and maximum values. The domain intervals are divided into a prespecified number of subintervals. Number and lengths of these subintervals are determined with intuition and visual inspection. The production and the attraction variables are divided into five, the friction variable is divided into six and the output variable, trips, is divided into 20 fuzzy sets. The fuzzy sets are labelled with numbered MFs representing low, moderate and high quantities roughly. For simplicity, the first and the last MFs are established as semi-trapezoidal and the others are set as triangular. Figure 4 illustrates the scaled fuzzy sets originally used in the study.

Generate fuzzy rule candidates from numerical data:

In this step, membership degrees $\left[\mu\left(x_{i}\right)\right]$ of each input-output point are evaluated, and then MFs having maximum degrees are assigned as a rule candidate. Suppose that membership degrees evaluated for any pair of data are indicated as following:

$$
\begin{aligned}
\mu_{\left(P_{i}, A_{i}, F_{i} ; T_{i}\right) \rightarrow} & {\left[P_{i}\left(0.7 \text { in } M F_{1} ; 0.4 \text { in } M F_{2}\right), A_{i}\left(0.8 \text { in } M F_{1} ; 0.2 \text { in } M F_{2}\right),\right.} \\
& \left.F_{i}\left(0.6 \text { in } M F_{1} ; 0.3 \text { in } M F_{2} ;\right), T_{i}\left(0.9 \text { in } M F_{2} ; 0.2 \text { in } M F_{3}\right),\right]
\end{aligned}
$$


Assigning the corresponding MFs with a maximum degree to a logical If-Then structure constitutes the $i$ th rule candidate as:

$$
\begin{aligned}
i^{\text {th }} \text { rule candidate } \rightarrow & I F P_{i} \text { is } M F_{1} \text { and } A_{i} \text { is } M F_{1} \text { and } F_{i} \text { is } M F_{1}, \\
& T_{i} \text { is } M F_{2}
\end{aligned}
$$

Select one desired rule among conflicting rules:

Three input structures, $P, A$ and $F$ with five, five and six fuzzy partitions, respectively, enable $150(5 * 5 * 6)$ different rule antecedents (IF part of a rule) with logical 'and' connections. However, the training data-set consists of 101,926 observed inputoutput pairs leading to a great number of conflicting rules: the rules having same antecedents but different consequents (THEN part of a rule). The traditional approach of the original Wang-Mendel method evaluates the strength of each rule candidate with multiplying their membership degrees, and then selects a final rule having the maximum strength. Since this approach does not satisfy our case due to high number of the same rule candidates, this step is processed in a different way: first, the weighted average of conflicting rule consequents are computed using their observed frequencies, then the nearest consequent part to the computed weighted average is selected as the final rule. For instance, if three different rule consequents are observed with various frequencies as in the following example:

$$
\begin{aligned}
\text { IF } P_{i} \text { is } M F_{1} \text { and } A_{i} \text { is } M F_{1} \text { and } F_{i} \text { is } M F_{1}, & \text { THEN } T_{\mathrm{i}} \text { is } M F_{2} \rightarrow \text { frequency } 25 \\
\text { THEN } T_{\mathrm{i}} \text { is } M F_{3} & \rightarrow \text { frequency } 35 \\
\text { THEN } T_{\mathrm{i}} \text { is } M F_{5} & \rightarrow \text { frequency } 20
\end{aligned}
$$

a final rule consequent (MF label number) can be selected computing their weighted average as in the following expression:

$n_{w a}=\frac{\left(n_{1} * f_{1}\right)+\left(n_{2} * f_{2}\right)+\ldots+\left(n_{k} * f_{k}\right)}{f_{1}+f_{2}+\ldots+f_{k}} \Rightarrow \frac{(2 * 25)+(3 * 35)+(5 * 20)}{25+35+20} \cong 3$

where, $n_{k}$ and $f_{k}$ represent the observed label number of a MF and corresponding frequency, and $n_{w a}$ represents the weighted average of the conflicting rule consequents. Then the final statement of the rule in the above example should end with $M F_{3}$, since the computed $n_{w a}$ equals approximately 3 .

Combine selected fuzzy rules and generate fuzzy rule base:

This step is the final step of an automated fuzzy rule-based learning procedure. Steps one through four are automated with a self-created programme written in the MATLAB environment. In this step, all single rules, selected in the previous step, are combined establishing the initial fuzzy rule base.

Check out fuzzy rule base and make a limited number of changes:

Approximately $95 \%$ of the rule base has been directly generated from numerical input-output pairs with the first four steps. Then, non-observed rules are extrapolated making an analogy to neighbourhood rules having similar antecedents. 
Table 1. An appearance from the constructed rule base.

\begin{tabular}{llc}
\hline Rule number & \multicolumn{1}{c}{ Antecedents } & Consequents \\
\hline Rule 1 & IF $P_{i}$ is $M F_{I}$ and $A_{i}$ is $M F_{I}$ and $F_{i}$ is $M F_{I}$ & THEN $T_{i}$ is $M F_{3}$ \\
Rule 2 & IF $P_{i}$ is $M F_{I}$ and $A_{i}$ is $M F_{2}$ and $F_{i}$ is $M F_{I}$ & THEN $T_{i}$ is $M F_{3}$ \\
Rule 3 & IF $P_{i}$ is $M F_{I}$ and $A_{i}$ is $M F_{3}$ and $F_{i}$ is $M F_{I}$ & THEN $T_{i}$ is $M F_{4}$ \\
Rule 23 & IF $P_{i}$ is $M F_{5}$ and $A_{i}$ is $M F_{3}$ and $F_{i}$ is $M F_{I}$ & THEN $T_{i}$ is $M F_{18}$ \\
Rule 24 & IF $P_{i}$ is $M F_{5}$ and $A_{i}$ is $M F_{4}$ and $F_{i}$ is $M F_{I}$ & THEN $T_{i}$ is $M F_{19}$ \\
Rule 25 & IF $P_{i}$ is $M F_{5}$ and $A_{i}$ is $M F_{5}$ and $F_{i}$ is $M F_{I}$ & THEN $T_{i}$ is $M F_{20}$ \\
Rule 48 & IF $P_{i}$ is $M F_{5}$ and $A_{i}$ is $M F_{3}$ and $F_{i}$ is $M F_{2}$ & THEN $T_{i}$ is $M F_{11}$ \\
Rule 49 & IF $P_{i}$ is $M F_{5}$ and $A_{i}$ is $M F_{4}$ and $F_{i}$ is $M F_{2}$ & THEN $T_{i}$ is $M F_{15}$ \\
Rule 50 & IF $P_{i}$ is $M F_{5}$ and $A_{i}$ is $M F_{5}$ and $F_{i}$ is $M F_{2}$ & THEN $T_{i}$ is $M F_{16}$ \\
Rule 148 & IF $P_{i}$ is $M F_{5}$ and $A_{i}$ is $M F_{3}$ and $F_{i}$ is $M F_{6}$ & THEN $T_{i}$ is $M F_{1}$ \\
Rule 149 & IF $P_{i}$ is $M F_{5}$ and $A_{i}$ is $M F_{4}$ and $F_{i}$ is $M F_{6}$ & THEN $T_{i}$ is $M F_{1}$ \\
Rule 150 & IF $P_{i}$ is $M F_{5}$ and $A_{i}$ is $M F_{5}$ and $F_{i}$ is $M F_{6}$ & THEN $T_{i}$ is $M F_{2}$ \\
\hline
\end{tabular}

Finally, the whole rule base has been checked logically and a limited number of rules $(10 \%)$ have been changed to improve the generalizability of the system. Table 1 indicates the latest appearance of the rule base.

After having established the MFs and the rule base, the next step is to select the implementation techniques. The implication procedure of the proposed FRBS for both training and testing purposes is described in the following steps:

Initialization: Initialize data and normalize input-output spaces into some appropriate range when it is required. Constitute the knowledge base.

Fuzzification: Compute fuzzy membership degrees of actual inputs using corresponding MFs: crisp into fuzzy values.

Inference: Combine fuzzy sets with logical operators with appropriate implication algorithm. Aggregate all outcomes to generate fuzzy output. In this step, both MaxMin and Max-Product techniques are tried and the Max-Product implication is selected.

Defuzzification: Convert aggregated fuzzy outputs into crisp outputs using a defuzzification method. In this step, the Centroid Defuzzification method has been selected and implied within various defuzzification techniques.

Graphical interpretation of this procedure is beneficial to understand the nature of fuzzy trip distribution modelling. The illustration in Figure 4 shows the original components and scales of the proposed FRBS design.

The proposed FRBS produces unconstrained trip interactions as output. Before using it for simulation purposes, we had to ensure that the results satisfy production and attraction constraints. Therefore, the results of the FRBS were adjusted with a row-column balancing process in each simulation of data sets. A numerical example of such a balancing process, which is similar to the well-known Furness Iterations (1965), can be seen in Easa (1993). 

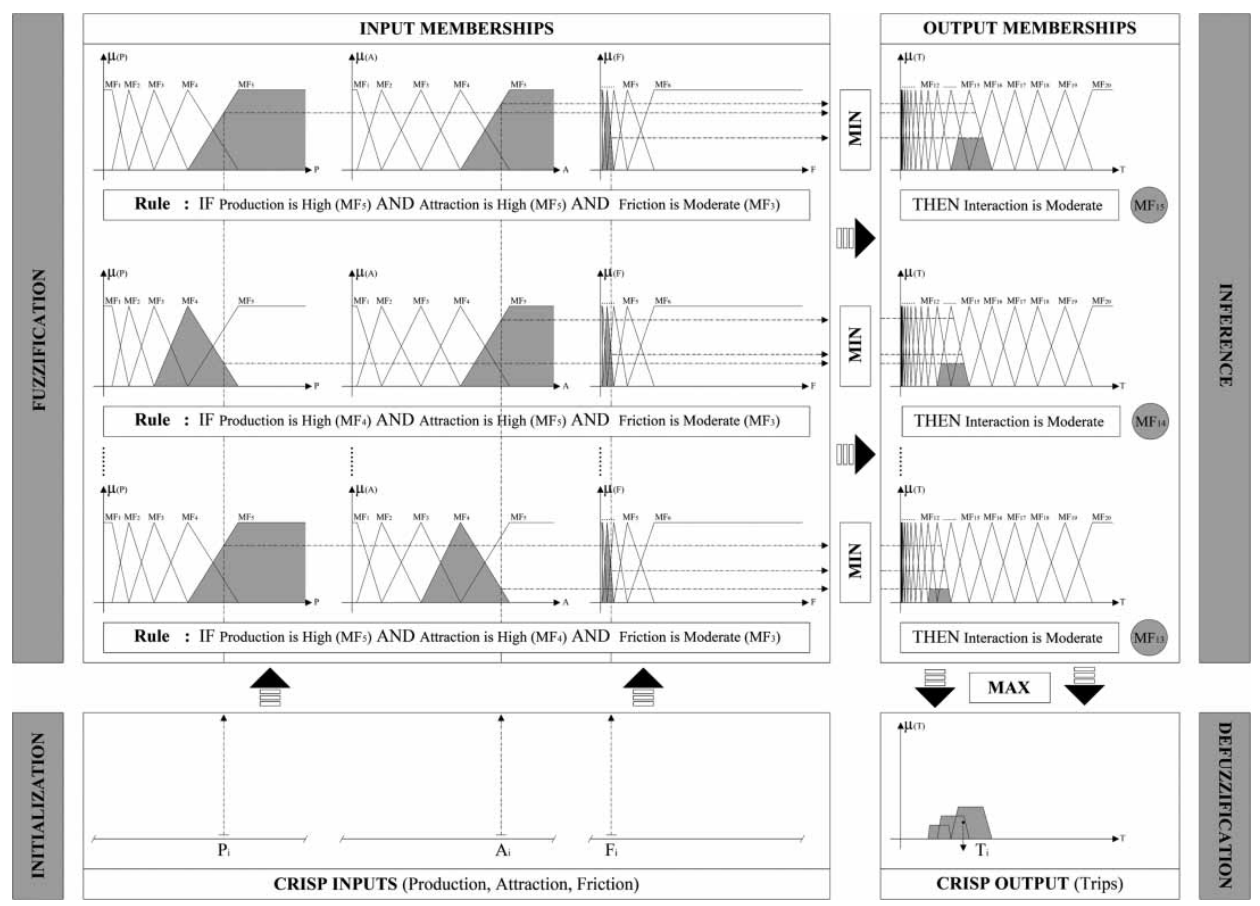

Figure 4. Graphical illustration of the proposed FRBS design.

\section{Modelling trip distribution with a GFRBS}

Main components of GFRBSs

FRBSs have an advantage of storing knowledge that is learned from the data itself or set up by an expert. However, they lack a self-learning feature. If the knowledge of a system is fixed and well-defined, it is easy to design an effective FRBS. On the other hand, an increase in the size and complexity of the knowledge base makes the design process of an optimum FRBS difficult. One of the recent approaches to eliminate this learning deficiency of fuzzy systems is to enhance them using GAs.

GAs are effective tools with acceptable solutions when exploring large search spaces in a reasonable time. First initiated by Holland (1975) and his colleagues, they are guided random search techniques which are primarily based on Darwin's principles of natural selection and the genetics branch of biology. In a GA process, genetic codes of individuals within a population evolve into a solution with the overriding principle of survival of the fittest. New generations produce new individuals with improved genetic codes via reproduction, crossover and mutation operators.

Typically a GA procedure can be identified with some main steps as: the creation of the initial population and the evaluation function; the determination of chromosome representation and selection function; and finally the set of parameters for genetic operators, reproduction and termination criteria. GAs can work well in a wide variety of engineering problems. They have also attracted considerable attention in a great number of disciplines as a methodology of search optimisation and learning, especially after the work of Goldberg (1989). 
As mentioned above, use of GAs in enhancing or optimizing fuzzy systems has started a new field of research area that is called GFSs. The most widely used types of GFSs are GFRBSs, which incorporate evolutionary techniques to achieve automatic generation or modification of FRBS knowledge base components (Cordon et al., 2001). Fuzzy MFs, rule base and even scaling functions can be stored in knowledge base of a fuzzy system. In designing a GFRBS, either part of or the entire knowledge base can be subject to optimisation by GAs. Figure 3, which was introduced earlier, shows the integration of GAs with FRBSs.

GFRBS designs generally fall into one of four categories: (1) use of GAs to tune the MFs under a given set of fuzzy rules; (2) use of GAs to specify the rule base with fixed MFs; (3) use of GAs to learn both the database and the rule base simultaneously; and (4) use of GAs to learn the database and the rule base sequentially. The present study deals with the second category: modifying or learning the rule base of a predefined FRBS.

Additionally, GAs can be used with various representations in genetic learning of a rule base. These representations generally follow two different approaches: (1) the 'chromosome $=$ set of rules', and the 'chromosome = rule' (Herrera, 2008). The first approach, also known as the Pittsburgh Approach (Smith, 1980), is selected as the genetic learning strategy of the proposed design. The Pittsburgh approach successfully solves the cooperation-versus-competition problem by evolving a population of rule bases instead of single rules. However, it brings a much greater computational burden which can be solved with improved genetic operators. The following section presents implementation and design issues for the proposed GFRBS in this regards. The reader is referred to Cordon et al. (2001) and Bodenhofer and Herrera (1997) for further reviews of the main types of GFRBS and related genetic learning strategies.

\section{The proposed GFRBS design}

The rule base of the previously introduced FRBS was constructed with a mixed procedure including both learning from examples and expertise. Here, the rule base of that FRBS is learnt completely from examples with the use of GAs. In other words, the proposed GA search for the best combination of rule consequents (MF labels) represented with grey circles in Figure 4 introduced earlier.

Initially, a simple GA (Goldberg, 1989) was developed with basic genetic operators and binary representation. However, due to the large combinatorial search space and huge amount of data, the convergence failed and some additional modifications have been introduced to improve the convergence performance of the algorithm. Keeping the main flow chart and its binary representation, several probabilistic and adaptive features were introduced to the genetic operators. The flow chart in Figure 5 indicates the main steps of the proposed GFRBS fully automated and programmed with MATLAB. A brief description of the whole procedure is given later.

Initialization: The initial population of the GA consists of randomly generated 20 binary chromosomes encoding the whole rule set. The chromosomes have 450 binary digits where three digits were assigned for each of the 150 rule consequents, meaning that a rule can end with one of eight $\left(2^{3}\right)$ alternatives. Actually, there are 20 output MFs in the proposed FRBS, however, a few shifting consequents are meaningful for each rule antecedent. Eventually, the maximum number of alternatives was restricted 


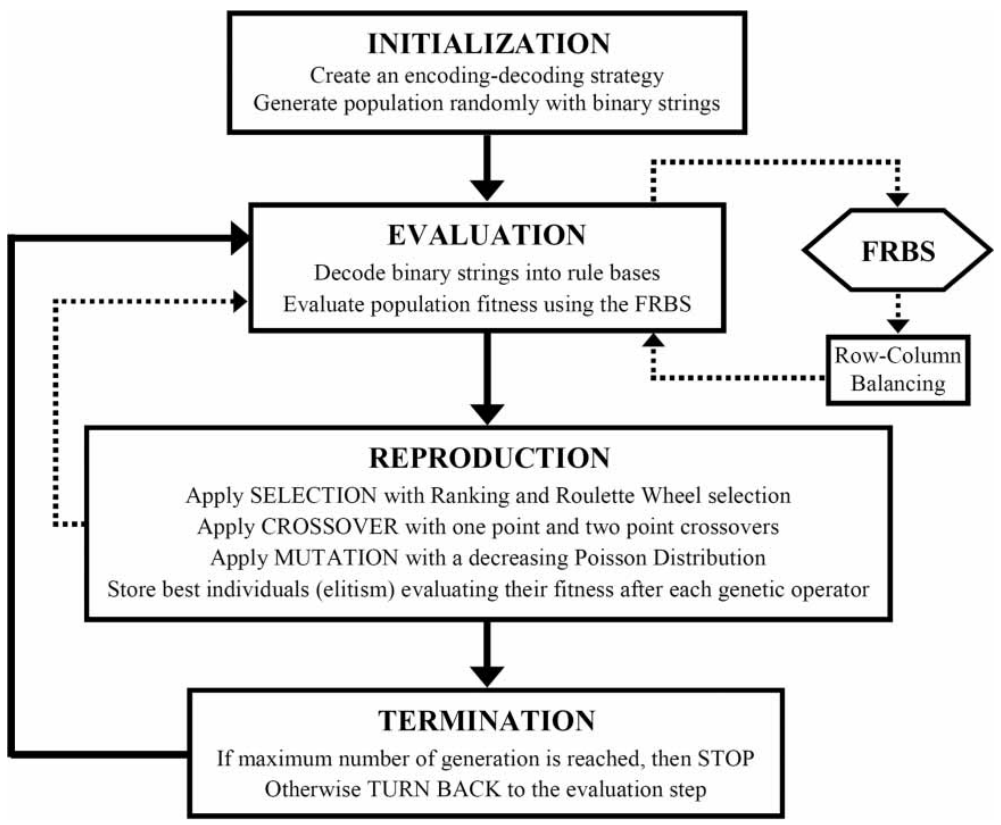

Figure 5. Flow chart of the proposed GFRBS design.

\begin{tabular}{|c|c|}
\hline $\begin{array}{l}\text { Rule } \\
\text { Number }\end{array}$ & $\begin{array}{c}\text { Antecedents } \\
\text { Input: Production-Attraction-Friction }\end{array}$ \\
\hline Rule 1 & IF $P_{i}$ is $M F_{l}$ and $A_{i}$ is $M F_{l}$ and $F_{i}$ is $M F_{l}$ \\
\hline Rule 2 & IF $P_{i}$ is $M F_{l}$ and $A_{i}$ is $M F_{2}$ and $F_{i}$ is $M F_{l}$ \\
\hline Rule 3 & IF $P_{i}$ is $M F_{l}$ and $A_{i}$ is $M F_{3}$ and $F_{i}$ is $M F_{l}$ \\
\hline ..... & $\ldots .$. \\
\hline Rule 23 & IF $P_{i}$ is $M F_{5}$ and $A_{i}$ is $M F_{3}$ and $F_{i}$ is $M F_{l}$ \\
\hline Rule 24 & IF $P_{i}$ is $M F_{5}$ and $A_{i}$ is $M F_{4}$ and $F_{i}$ is $M F_{l}$ \\
\hline Rule 25 & IF $P_{i}$ is $M F_{5}$ and $A_{i}$ is $M F_{5}$ and $F_{i}$ is $M F_{l}$ \\
\hline Ru... 148 & IF. $P$ is $M F_{\text {s }}$ and $A$ is $M F_{3}$ and $F$ is $M F_{\text {, }}$ \\
\hline Rule 149 & IF $P_{i}$ is $M F_{5}$ and $A_{i}$ is $M F_{4}$ and $F_{i}$ is $M F_{6}$ \\
\hline Rule 150 & IF $P_{i}$ is $M F_{5}$ and $A_{i}$ is $M F_{5}$ and $F_{i}$ is $M F_{6}$ \\
\hline
\end{tabular}

\begin{tabular}{|c|c|c|c|c|c|c|c|c|}
\hline $\begin{array}{l}\text { Consequents } \\
\text { Output: Trips }\end{array}$ & \multicolumn{8}{|c|}{$\begin{array}{l}\text { Conflicting } \\
\text { Rule Pool }\end{array}$} \\
\hline \multirow{4}{*}{$\begin{array}{l}\text { THEN } T_{i} \text { is } M F \rightarrow \\
\text { THEN } T_{i} \text { is } M F \rightarrow \\
\text { THEN } T_{i} \text { is } M F \rightarrow \\
\ldots . .\end{array}$} & 1 & 2 & 3 & 4 & 5 & 6 & & \\
\hline & 2 & & & 5 & 6 & 7 & & 9 \\
\hline & 3 & 4 & 5 & 6 & 7 & 8 & & 10 \\
\hline & & & & & & & & \\
\hline \multirow{4}{*}{$\begin{array}{l}\text { THEN } T_{i} \text { is } M F \rightarrow \\
\text { THEN } T_{i} \text { is } M F \rightarrow \\
\text { THEN } T_{i} \text { is } M F \\
\ldots . .\end{array}$} & & 14 & 15 & 16 & 17 & 18 & & 20 \\
\hline & & 17 & 18 & 18 & 19 & 19 & 20 & 20 \\
\hline & & 19 & 19 & 19 & 20 & 20 & & 20 \\
\hline & & & & & & & & \\
\hline \multirow{3}{*}{$\begin{array}{l}\text { THEN } T_{i} \text { is } M F \rightarrow \\
\text { THEN } T_{i} \text { is } M F \rightarrow \\
\text { THEN } T_{i} \text { is } M F \rightarrow\end{array}$} & & & 2 & 2 & & 3 & & 4 \\
\hline & & 1 & 2 & 2 & 3 & 3 & & 4 \\
\hline & & 1 & 2 & 2 & 3 & 3 & 4 & 4 \\
\hline
\end{tabular}

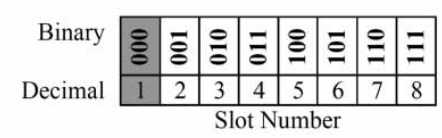

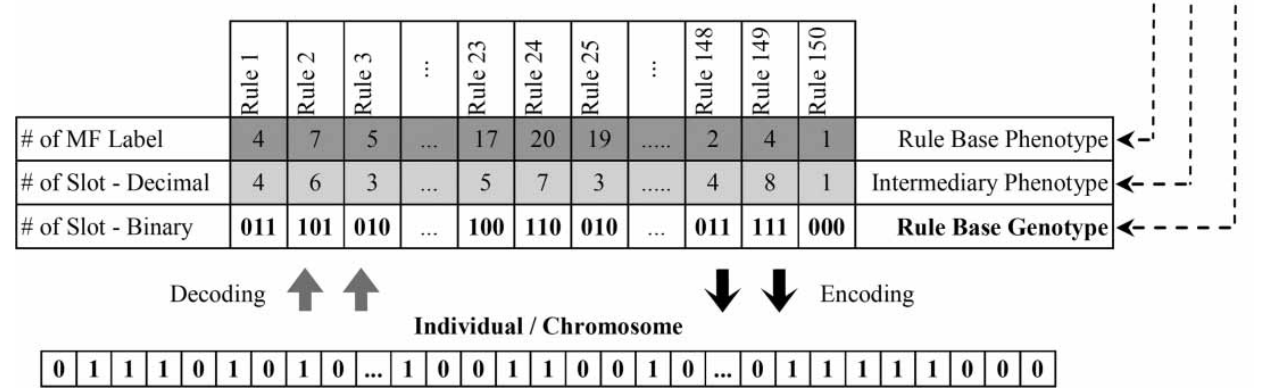

Figure 6. Graphical representation of encoding-decoding strategy. 
to eight in order to reduce the search space and save time. The alternatives were obtained from available knowledge according to the conflicting rules of the FRBS design. The most frequently observed or most probable two, four or eight rule consequents were identified for each rule antecedent and collected in a pool. With this rule pool, 45 rules can end with one of two, 65 rules can end with one of four, and 40 rules can end with one of eight MF alternatives constituting $6.4^{*} 10^{88}\left(2^{45 *} 4^{65 *} 8^{40}\right)$ possible rule sets. Figure 6 shows the whole encoding-decoding strategy including a view from the rule base and the rule pool.

Evaluation: In the evaluation step, the binary strings were decoded into the rule sets to run the FRBS. Then output values of the FRBS for each of the rule set were compared with the actual values. The mean square error (MSE) was used as the fitness (error) function:

$$
\mathrm{MSE}=\frac{\sum_{i=1}^{N}\left(T_{i}^{0}-T_{i}\right)^{2}}{N}
$$

where, $N$ is the number of data pairs, $T_{i}^{0}$ is the number of observed trips, and $T_{i}$ is the number of estimated trips. The FRBS estimated trips were also balanced to satisfy the constraints before making the comparisons.

Reproduction: Generally in GAs, successive generations of the population should base on transferring the best chromosomes to next generation (selection), and then improving them with gene exchanges (crossover) and gene alterations (mutation). The proposed GA was designed with this three step evolution process described as follows:

- Selection: In this step 'successful' chromosomes, the parents, were copied to a mating pool, then selected for crossover and mutation according to some measure of their fitness. There are a number of ways to choose the parent population. A mixed procedure was implemented in order to improve the convergence performance: First, a ranking was applied, in which chromosomes are ranked and assigned proportions only on their rank orders, not on their absolute fitness. Then mating parents were selected with a biased roulette-wheel for recombination. The slots of the wheel were divided according to the ranking proportions determined with a power function (see Figure 7). Apart from these, when generating new populations with genetic selection, crossover and mutation operators an elitist strategy was developed. The few best chromosomes $(10 \%$ of the total population) of the former generations were directly copied to the next generations through genetic operators. This strategy significantly improved the GA's performance preventing it from loss of any good solutions.

- Crossover: In the crossover step, new chromosomes (offspring) were created by recombining two parent chromosomes with a certain probability $(0.8)$. Classical one-point or two-point crossovers were employed to the parent chromosomes with equal probability. In the one-point crossover, the algorithm chooses a point at random, called the crossover point, and exchanges the contents to the right of this point; in the two-point crossover, the algorithm chooses two points, and exchanges the contents between these points. Figure 7 indicates an illustration of the adopted crossover technique used in the GA. 

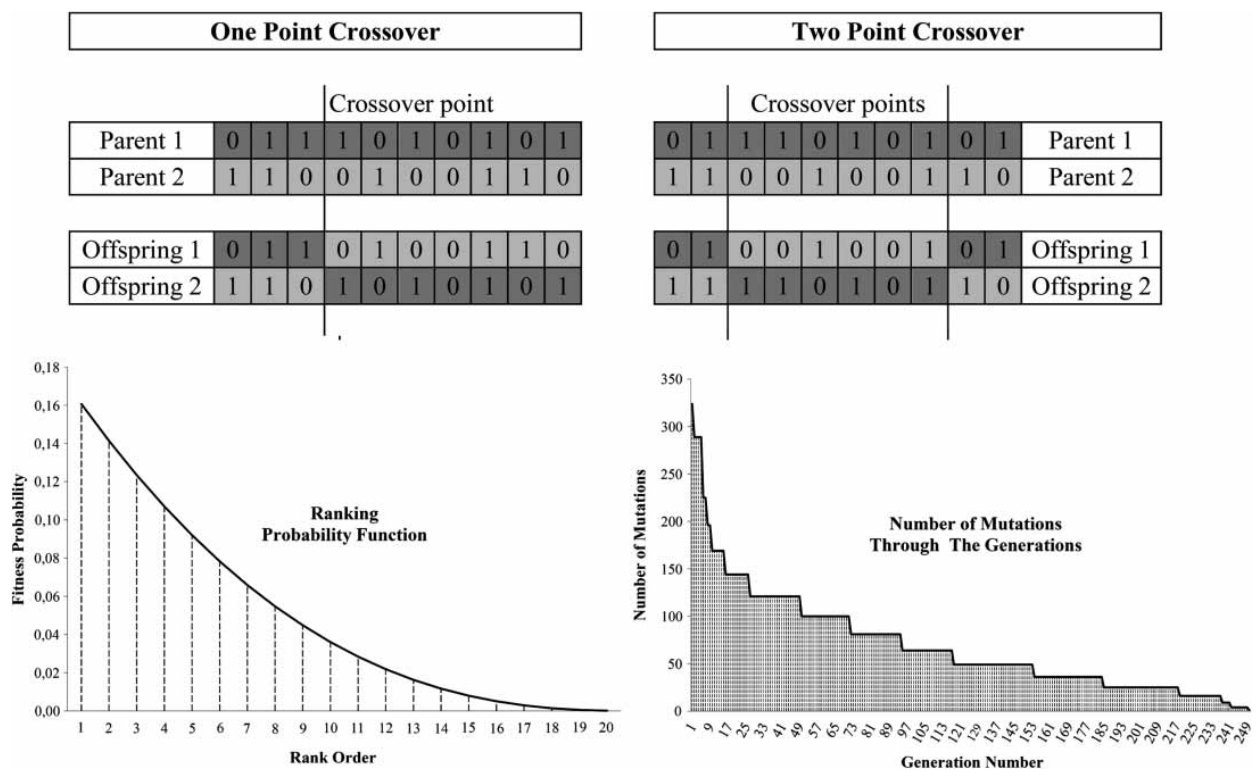

Figure 7. Illustrations of crossover, ranking probability function and number of mutations through the generations.

- Mutation: In order to achieve faster results and prevent the algorithm from a premature convergence, two components were introduced to the classical mutation operator. First, the assumption of a constant probability of mutation in each generation is abandoned in favour of an adaptive one. With high mutation probability at the start up, the population attacked to get out of from local optima; and with low probability at the end, the population resembled each other to find out small improvements. Second, the simple bit-flip mutation operator has been replaced with a probabilistic (Poisson distribution) mutation operator. This approach is an efficient and time-saving alternative of simple bit-flip mutation. In this approach, the average number of mutations $(\lambda=72)$ in each generation is determined automatically, multiplying the population size (20), chromosome length (450) and mutation probability (0.008). Then the number of mutations in various generations is determined with a decreasing Poisson distribution at the start up. Finally, only in that number of randomly selected bits in the whole population is changed (inverted) through the generations. A graph that shows number of mutations through the generations can be seen again in Figure 7.

Termination: Any early stopping of the GA was not seen necessary as the termination criteria. We rather limited the evolution of the population up to 250 maximum generations.

With the above procedure, the GFRBS design successfully converged to the best solution. Then it was used for simulation purposes with the optimized rule base. Figure 8 indicates both the progress of the population average and the best individual. 


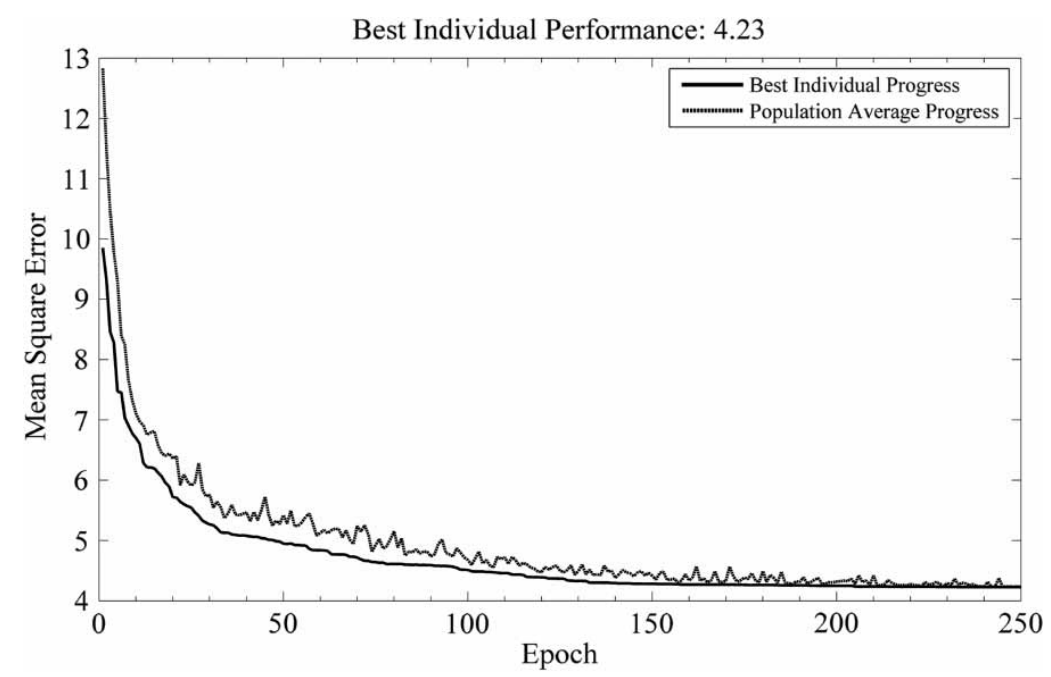

Figure 8. Convergence of the GFRBS design.

\section{Benchmark models}

The DCGM, introduced earlier, was selected as the first benchmark model. A NNbased Trip Distribution Model (NNTDM) was established as the second benchmark. Even though debate continues, NN-based distribution models have a history of successful use, and it is appropriate to establish a NN-based model and compare its overall performance with the proposed FRBS and GFRBS designs. The next sections of this paper cover calibration, training, and implementation issues for these benchmark models.

\section{Doubly constrained gravity model (DCGM)}

Many studies have suggested statistical or numerical computational procedures to calibrate friction parameters for gravity models (Hyman, 1969; Wilson, 1970; Evans, 1971; Sen and Soot, 1981; Dickey, 1983; Sen, 1986). Among these procedures, statistical weighted least squares (WLS) and maximum likelihood (ML) estimations, and numerical TLD-based estimation are found efficient for our analysis. A brief description of these calibration techniques can be given as follow:

(1) ML estimation maximizes the likelihood function of a theoretical Poisson distribution of interactions and is described in Sen (1986) and Fotheringham and O’kelly (1989);

(2) WLS Estimation is based on the odds ratio technique and logarithmic transformation proposed by Sen and Soot (1981) for rectangular interaction matrices; and

(3) TLD-based Estimation uses a simple line search algorithm to find the best value of $\beta$. First, trip matrices are estimated using $\beta$ values in a search interval $(0-4)$; the TLD of the matrices are then computed, and observed and estimated TLDs are compared with their root mean squared error (RMSE). 
The $\beta$ value with the lowest RMSE score was determined as the friction parameter. Similar procedures, but for discrete cases, can be seen in Dickey (1983) and Transportation Research Board (1998).

Regression-based techniques and ML approaches have well-known desirable statistical properties, and they have consistently proven their calibration abilities. The TLD-based numerical approaches also have attractive properties. They enable a better understanding of system behaviour, especially in instances when a great number of inter-zonal trips are missing. The three selected principally distinct calibration procedures gave considerably different outputs, and all of them are included in performance comparison and model evaluation. All calibration procedures and algorithms are realized using MATLAB, and the friction parameters of each procedure are computed using the training data-set. The use of a power-cost function as friction factor produces considerably more accurate estimations for the Istanbul case. Therefore, only the results produced by the power-cost function are presented in the analysis.

\section{Neural network-based trip distribution model (NNTDM)}

The logical structure of an NNTDM involves three inputs (production, attraction and friction) and one output (interactions) as in the traditional trip distribution

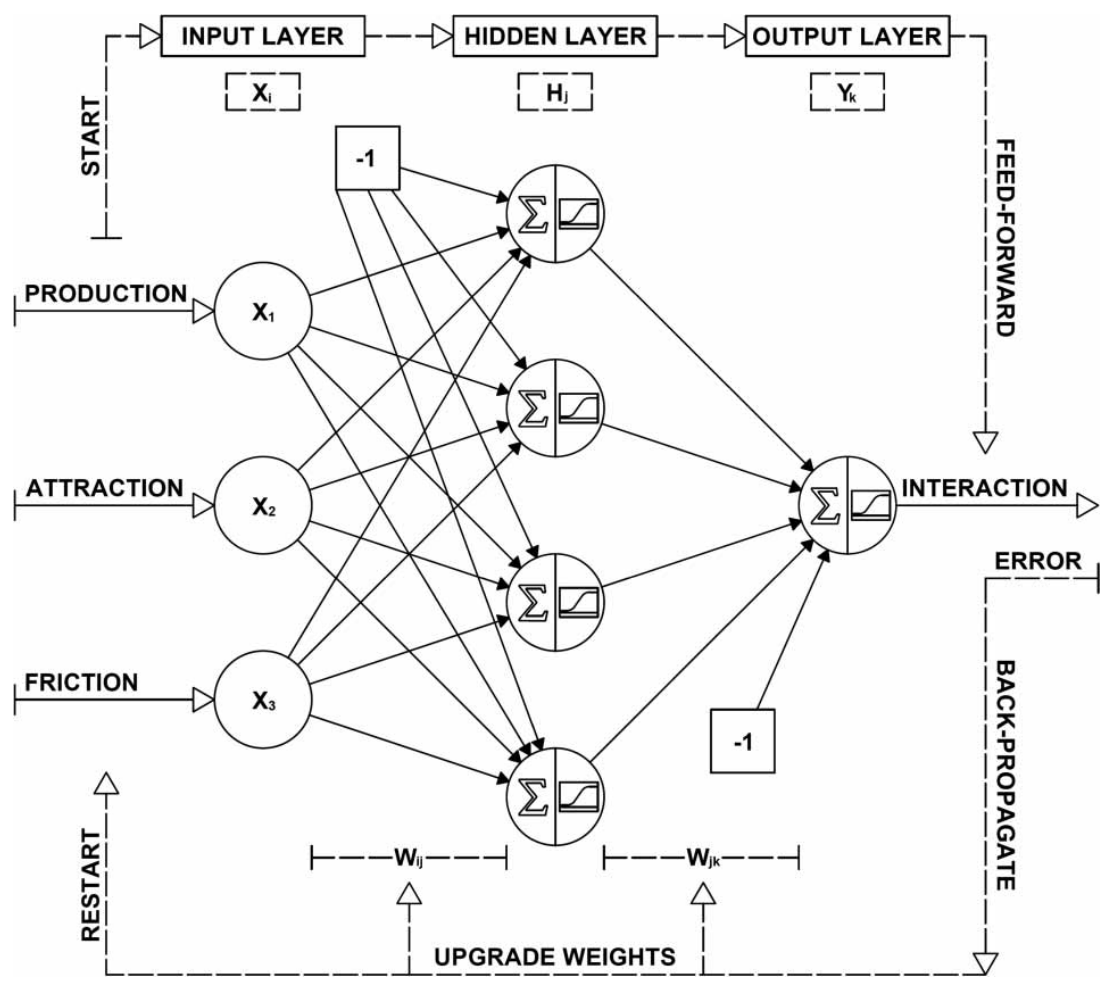

Figure 9. An illustration of NNTDM - A three-layer feed-forward neural network with error back-propagation. 
problem. There are many types of NN models with various functionality and architectures. However, the subject of the previous studies cited earlier and the present paper is a multilayer feed-forward network with an (error) back-propagation training algorithm.

A general illustration of a NN-based distribution model, also used in this study, is presented in Figure 9. The number of neurons in the hidden layers, the number of hidden layers, type of transfer functions, and learning algorithms as well as the number of inputs can be changed for proper use. In-depth explanation of a multilayer feed-forward back-propagation network with its mathematical expressions can be seen in Munakata (2008) and Haykin (1999).

Defining the variables and the main architecture of the network is the first step of the NN modelling. The other steps include developing a strategy to avoid overtraining (where the network learns incorrect information/noise, instead of the general pattern) and selecting appropriate training styles, activation functions, learning algorithms and parameter values. In order to prevent the network from over-training and to obtain the best generalization performance, the training data-set was further separated randomly into two: $80 \%$ for training the network and $20 \%$ for crossvalidation. As in common practice, other network configuration and training issues are processed by a trial-and-error selection. The complete process was realized in the MATLAB programming environment using the Neural Network Toolbox (see Demuth, Beale and Hagan, 2009). The trained network is then used to simulate datasets and to produce unconstrained trip interactions. Finally, a balancing process was applied to predicted flows as in the FRBS design in order to satisfy the trip constraints. Table 2 indicates the experimental and selected cases for network

Table 2. NNTDM implementation issues: experimented and selected cases.

\begin{tabular}{|c|c|c|}
\hline Implementation issues & Experimented cases & Selected cases \\
\hline Normalization technique & $\begin{array}{l}\text { - Z-score normalization } \\
\text { - Min-max normalization }\end{array}$ & 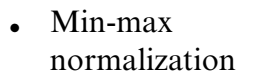 \\
\hline $\begin{array}{l}\text { Number of hidden layer } \\
\text { neurons }\end{array}$ & $\begin{array}{l}\text { - } 3-6-9-12-15 \\
\text { - } 20-25-30-40\end{array}$ & - 9 \\
\hline $\begin{array}{l}\text { Activation function of } \\
\text { hidden layer }\end{array}$ & $\begin{array}{l}\text { - Hyperbolic tangent function } \\
\text { - Logistic sigmoid function }\end{array}$ & - Logistic sigmoid \\
\hline $\begin{array}{l}\text { Activation function of } \\
\text { output layer }\end{array}$ & $\begin{array}{l}\text { - Hyperbolic tangent function } \\
\text { - Logistic sigmoid function } \\
\text { - Linear function }\end{array}$ & - Logistic sigmoid \\
\hline Training style & $\begin{array}{l}\text { - Batch training } \\
\text { - Incremental training }\end{array}$ & - Batch training \\
\hline Learning algorithm & $\begin{array}{l}\text { - Gradient descent with learning rate } \\
\text { - Gradient descent with adaptive learning } \\
\text { rate and momentum term } \\
\text { - Levenberg-marquardt }\end{array}$ & $\begin{array}{l}\text { - Levenberg- } \\
\text { marquardt }\end{array}$ \\
\hline Performance measure & $\begin{array}{l}\text { - } \quad \text { Mean squared/absolute error } \\
\text { - } r \text { square }\end{array}$ & $\begin{array}{l}\text { - } \text { Mean squared } \\
\text { error } \\
\text { - } r \text { square }\end{array}$ \\
\hline Termination criteria & $\begin{array}{l}\text { - Maximum epochs } \\
\text { - Validation performance }\end{array}$ & $\begin{array}{l}\text { - Validation } \\
\text { performance }\end{array}$ \\
\hline
\end{tabular}




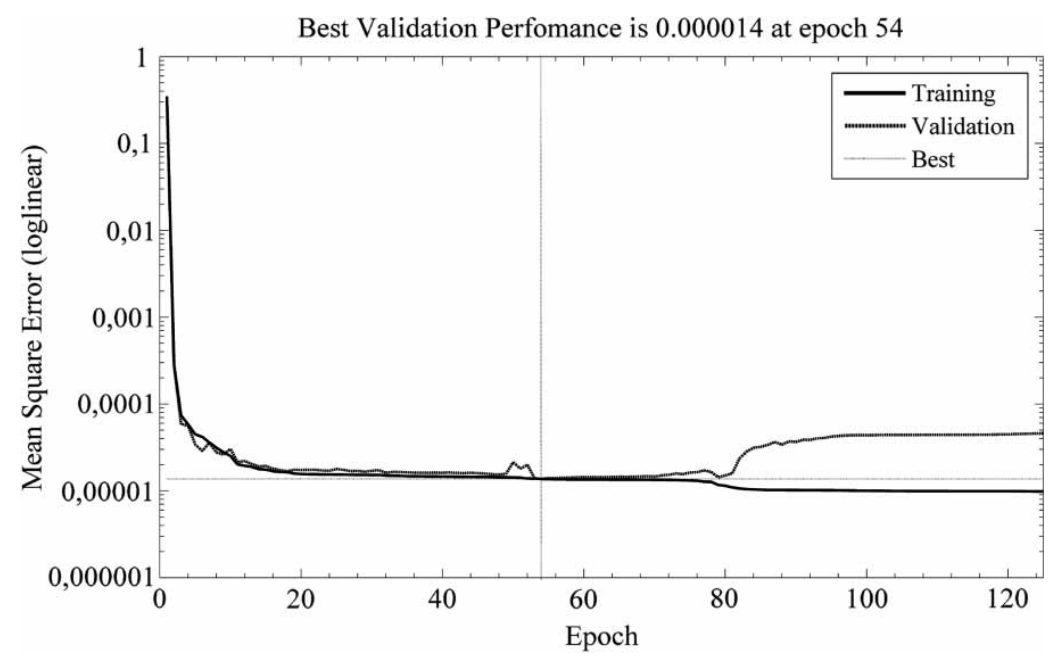

Figure 10. NNTDM back-propagation training with Levenberg-Marquardt learning.

training, and Figure 10 shows the convergence of the network with the LevenbergMarquardt learning algorithm.

\section{Performance measure and goodness-of-fit statistics}

Over the years, various goodness-of-fit statistics have been proposed to measure the accuracy of trip distribution model estimations. Four are found to be more representative in measuring the accuracy levels of models: (1) Standardized Root Mean Squared Error (SRMSE), (2) Coefficient of Determination $\left(R^{2}\right)$, (3) Trip Length Distribution Root Mean Squared Error (TLD RMSE) and (4) Mean Travel Cost Error (MTCE). Extensive reviews for these statistics can be found in Smith and Hutchinson (1981), Knudsen and Fotheringham (1986) and Fotheringham and Knudsen (1987). The following equations give mathematical expressions of the selected goodness-of-fit statistics:

$$
\begin{aligned}
\mathrm{SRMSE} & =\sqrt{\frac{\sum_{i j}\left(T_{i j}^{0}-T_{i j}\right)^{2}}{I \times J}} / \frac{\sum_{i j}\left(T_{i j}^{0}\right)}{I \times J} \\
R^{2} & =1-\frac{\sum_{i j}\left(T_{i j}^{0}-T_{i j}\right)^{2}}{\sum_{i j}\left(T_{i j}^{0}-\bar{T}^{0}\right)^{2}} \\
\mathrm{TLD}-\mathrm{RMSE} & =\sqrt{\frac{\sum_{i=1}^{n}\left(F_{i}^{0}-F_{i}\right)^{2}}{n}} \\
\mathrm{MTCE} & =\left(\frac{\sum_{i j}\left(T_{i j}^{0} \times C_{i j}\right)}{\sum_{i j} T^{0}}\right)-\left(\frac{\sum_{i j}\left(T_{i j} \times C_{i j}\right)}{\sum_{i j} T}\right)
\end{aligned}
$$

where,

$T_{i j}^{0}$ is the number of observed trips from zone $i$ to zone $j$, 
$T_{i j}$ is the number of estimated trips from zone $i$ to zone $j$,

$\bar{T}^{0}$ is the mean of the observed trips,

$I$ is the number of origins,

$J$ is the number of destinations,

$\Sigma \bar{T}^{0}$ is the total number of observed trips,

$\Sigma \bar{T}$ is the total number of estimated trips,

$C_{i j}$ is the travel cost/time between origin $i$ and destination $j$,

$F_{i}^{0}$ is the observed TLD percentage for the ith time interval,

$F_{i}$ is the estimated TLD percentage for the $i t h$ time interval, and

$n$ is the number of time intervals associated with trip length frequencies.

\section{Empirical findings}

This section presents the goodness-of-fit statistics of the previously introduced trip distribution models: a DCGM with Maximum Likelihood (DCGM ML) estimation, a Doubly Constrained Gravity Model with Weighted Least Squares (DCGM WLS) estimation, a Doubly Constrained Gravity Model with Trip Length Distribution (DCGM TLD)-based estimation, a NN-based Trip Distribution Model (NNTDM), a FRBS design and a GFRBS design. In the beginning, simulations for each of the training, testing and whole data-sets were produced and their performance in each case was measured with the above mentioned goodness-of-fit statistics. Finally, the performance of the models were measured further with respect to the results of district-based aggregation and trip share comparisons among intra-zonal vs. interzonal, intra-district vs. inter-district and bridge crossing vs. not bridge crossing trips.

Table 3. Model results - goodness-of-fit statistics.

\begin{tabular}{|c|c|c|c|c|c|c|}
\hline $\begin{array}{l}\text { Goodness-of-fit } \\
\text { statistics }\end{array}$ & $\begin{array}{l}\text { DCGM } \\
\text { ML Est. }\end{array}$ & $\begin{array}{c}\text { DCGM } \\
\text { WLS Est. }\end{array}$ & $\begin{array}{c}\text { DCGM TLD } \\
\text { Based Est. }\end{array}$ & NNTDM & $\begin{array}{l}\text { FRBS } \\
\text { design }\end{array}$ & $\begin{array}{c}\text { GFRBS } \\
\text { design }\end{array}$ \\
\hline \multicolumn{7}{|l|}{ Training results } \\
\hline SRMSE & 4.17 & 4.16 & 4.29 & 4.75 & 4.10 & 3.42 \\
\hline$R^{2}$ & 0.86 & 0.86 & 0.85 & 0.82 & 0.87 & 0.90 \\
\hline TLD RMSE & 0.10 & 0.18 & 0.07 & 0.11 & 0.11 & 0.07 \\
\hline MTCE & -2.77 & -1.65 & -4.18 & -4.14 & -4.29 & -1.07 \\
\hline \multicolumn{7}{|l|}{ Testing results } \\
\hline SRMSE & 4.25 & 4.34 & 4.27 & 4.42 & 4.15 & 3.91 \\
\hline$R^{2}$ & 0.78 & 0.78 & 0.77 & 0.75 & 0.78 & 0.81 \\
\hline TLD RMSE & 0.15 & 0.22 & 0.11 & 0.12 & 0.10 & 0.05 \\
\hline MTCE & -2.89 & -1.71 & -4.39 & -4.19 & -4.25 & -0.85 \\
\hline \multicolumn{7}{|c|}{ Whole data results } \\
\hline SRMSE & 4.88 & 5.10 & 4.82 & 5.02 & 4.56 & 4.30 \\
\hline$R^{2}$ & 0.77 & 0.77 & 0.77 & 0.75 & 0.79 & 0.82 \\
\hline TLD RMSE & 0.17 & 0.26 & 0.11 & 0.13 & 0.09 & 0.08 \\
\hline MTCE & -2.68 & -1.44 & -4.33 & -4.30 & -3.19 & -0.33 \\
\hline \multicolumn{7}{|c|}{ District-based results } \\
\hline SRMSE & 0.78 & 0.72 & 0.88 & 1.04 & 0.86 & 0.63 \\
\hline$R^{2}$ & 0.95 & 0.95 & 0.94 & 0.92 & 0.95 & 0.97 \\
\hline
\end{tabular}


The training results reported in Table 3 show the learning capacity of the implemented trip distribution models. As an overall evaluation, it can be stated that the GFRBS design demonstrated a certain superiority for almost all goodness-of-fit statistics, followed by the DCGMs and the FRBS design. The DCGM ML estimation was good for both micro- and macro-level statistics, whereas the DCGM WLS estimation was good at the micro level, and the DCGM TLD-based estimation was good for macro level statistics. The FRBS design achieved better results when the micro-level statistics are taken into account. It has the second best scores for SRMSE and $R^{2}$ but the second worst scores with the MTCE and TLD RMSE. Finally, the NNTDM obtained unexpected results in the training phase with the lowest SRMSE and $R^{2}$ scores. The reason for this can be that one of its implementation procedures was subject to row-column balancing, and was stopped to train before a possible over-training problem occurred.

The testing results of the models are more important than the training results. They represent the predictive ability of the models better, and give an idea of the generalizable performance of the trained models. According to the testing scores shown in Table 3, the GFRBS design outperforms all the other models. In comparison to the training results, the GFRBS design recorded a small reduction in SRMSE and $R^{2}$ scores and improved MTCE and TLD RMSE scores. This is a desired and expected situation that the GFRBS design has successfully learned the macro behaviour of the analyzed system. A similar observation is valid for the FRBS design. On the contrary to training, the FRBS design showed a better performance than DCGMs in the testing case. The FRBS design achieved the second best performance with respect to the three important statistics - SRMSE, $R^{2}$ and TLD RMSE scores. Apart from these, it can be said that the DCGM ML and DCGM TLD-based estimations preserved their performance and produced fairly good predictions in the testing case. However, the DCGM WLS estimation was worse especially when its TLD RMSE score is taken into account. The NNTDM model scores come close to the other models in the testing case, its SRMSE score decreased and its TLD RMSE score remained nearly the same. This is meaningful since it shows that the over-training strategy has worked well with the NNTDM.

The GFRBS design has again achieved the best scores when the models are simulated with the whole data-set. It obtained SRMSE, $R^{2}$, TLD RMSE and MTCE scores that are consistent with the training and testing cases. The FRBS design showed the second best performance in whole data simulation, especially with the SRMSE, $R^{2}$ and TLD RMSE scores. The DCGM ML and DCGM TLD-based estimation followed the FRBS design with a similar performance and fairly good predictions. The DCGM WLS estimation showed a worsening performance in whole data simulation; on the contrary to training and testing, its performance is poorer than the NNTDM with the worst TLD RMSE and SRMSE scores.

As it can be observed in the majority of previous cases, the proposed GFRBS design produced superior predictions. The FRBS design, the DCGM TLD-based estimation and the DCGM ML estimation in turn followed the GFRBS design. They also achieved high levels of accuracy and good levels of generalizability after the GFRBS design. The DCGM WLS estimation and the NNTDM did not show the expected performance; the performance of the DCGM WLS estimation especially decreased in both the testing and whole data-set simulations. 

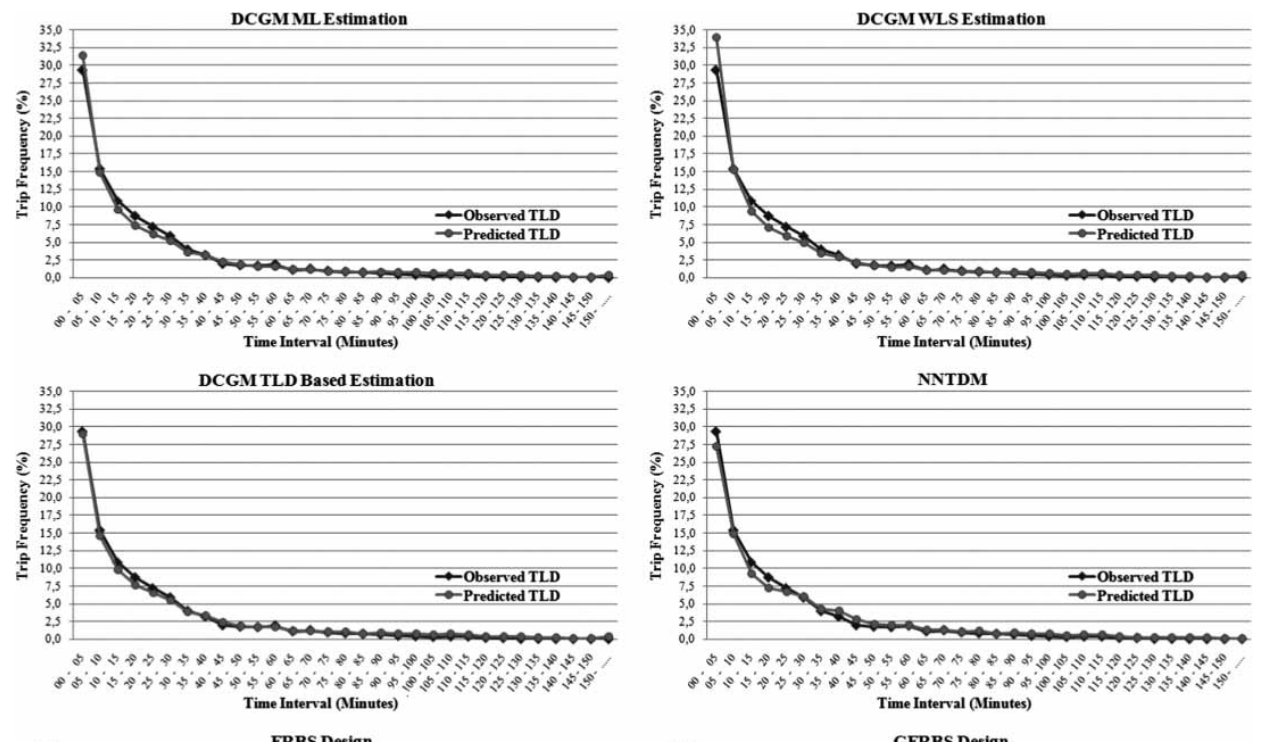

Time Interval (Minutes)
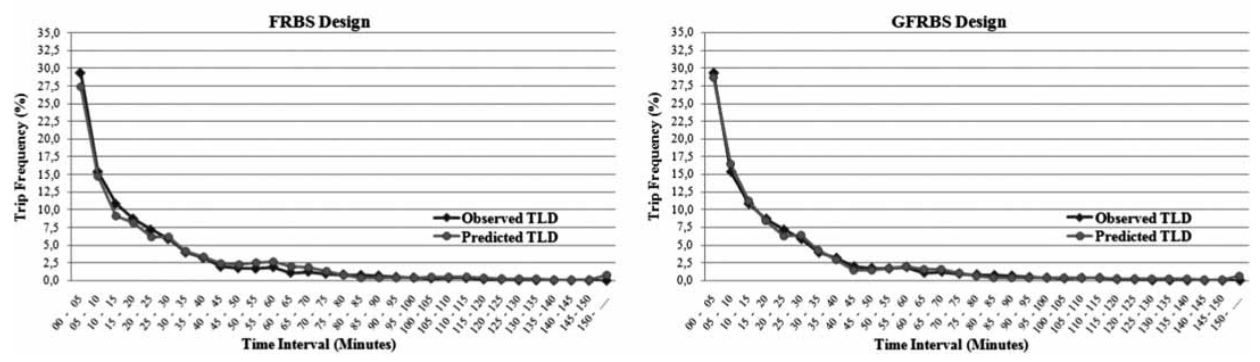

Figure 11. Observed-predicted TLDs - training data set.

Apart from these, as indicated with the graphical analysis of the TLDs in Figures 11 and 12, all the models captured the general trend of the observed TLD, except for the DCGM WLS estimation. In particular, the GFRBS design and the DCGM TLD-based estimation provided a near perfect fit to the observed TLDs. In addition, when the regression plots of the models - shown in Figures 13 and 14 - are analyzed, it can be said that all models showed a slight tendency to under-predict larger flows and over-predict smaller flows. This can be explained by the general structure of the observed flow matrix that mostly involves a small amount of flows. The GFRBS design and the DCGM WLS estimation is still successful in this respect. They produced considerably good predictions with a slope score close to unity.

The performance of the models was further tested with a district-based aggregation of trip interchanges. All the model results for TAZs were aggregated within the corresponding 31 districts in Istanbul metropolitan area. Then observed and predicted flows for the districts were compared using two micro level goodnessof-fit statistics as shown in Table 3. As in previous cases, the GFRBS design outperformed the other models. In general all models achieved good results, having $R^{2}$ scores of at least 0.92 . 

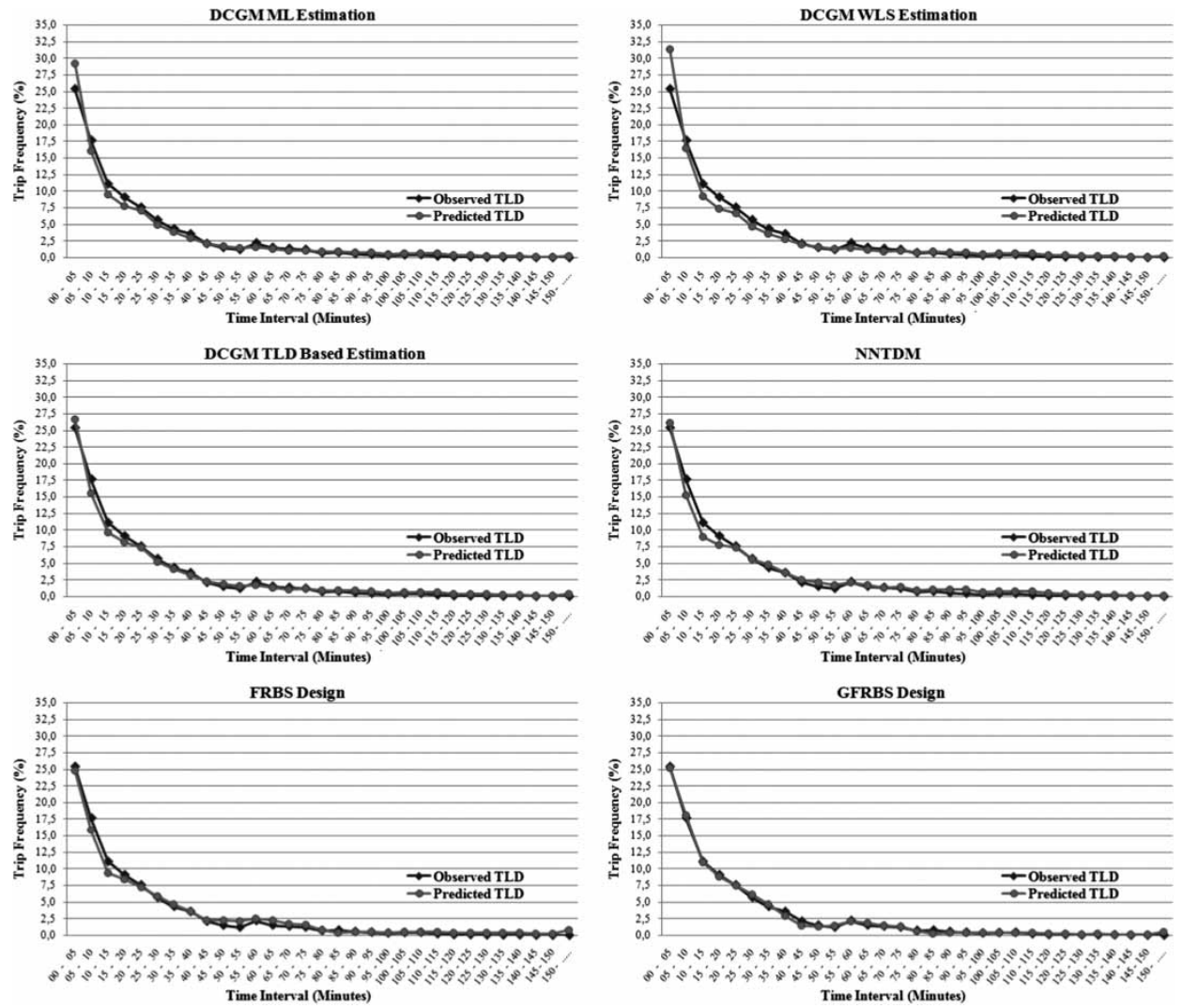

Time Interval (Minutes)

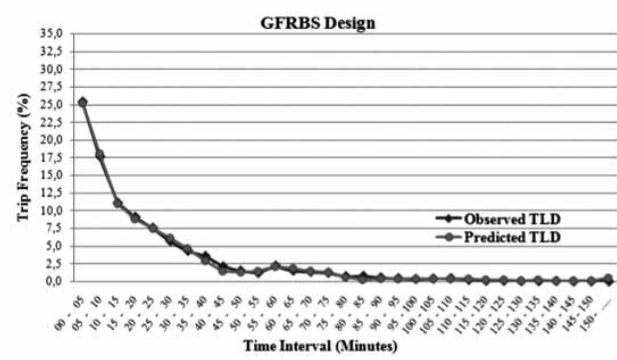

Figure 12. Observed-predicted TLDs - testing data set.

Finally, the spatial distribution of modelled trip interchanges was further measured with a trip share comparison. Observed and modelled trip shares among intra-zonal vs. inter-zonal, intra-district vs. inter-district and bridge crossing vs. not bridge crossing trips were measured. The results are shown in Table 4 . Nearly all the models successfully estimated trip shares with an error range of approximately $10-15 \%$ in most cases. Surprisingly, the DCGM WLS estimation outperformed the other models. We had mandatorily seeded the trip matrix with a very small number before calibrating the DCGM WLS estimation. The spatial distribution of the modelled trip interchanges could be affected by this implementation leading to a better performance of the model with respect to trip share comparison.

\section{Conclusions}

The general aim of this study was to set out a fuzzy and a GFS to estimate intra-city passenger flows, and thus contribute to the literature representing their potential use in trip distribution modelling. For this purpose, a simple Mamdani-type FRBS was developed to estimate trip interchanges in the Istanbul metropolitan area. Its rule base and fuzzy partitions were constructed with a mixed procedure including both learning from examples and expertise. Aggregate variables of the traditional trip 

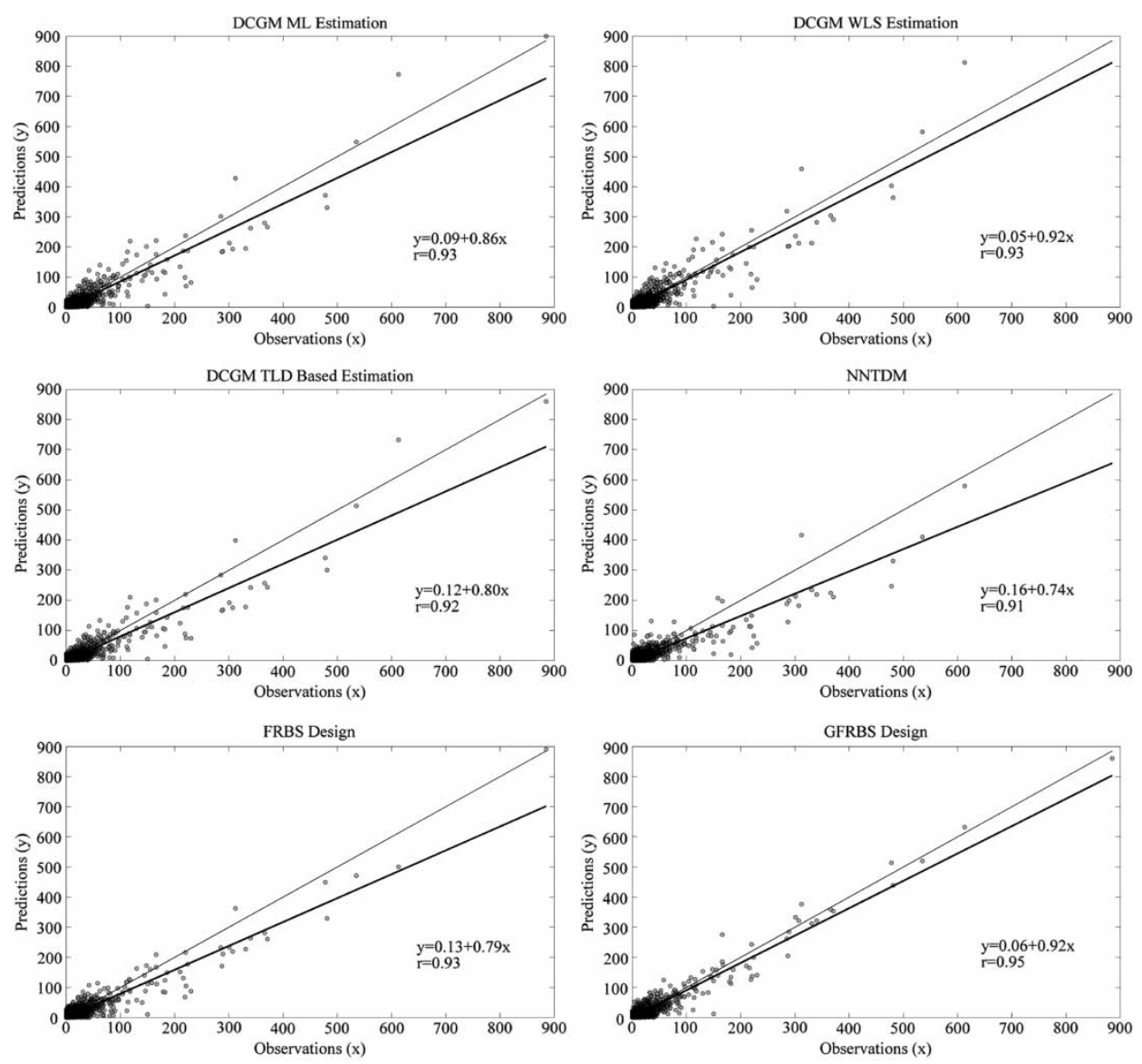

Figure 13. Regression plots - training data set.

distribution problem (production, attraction and friction) were used as inputs. The rule base of the proposed FRBS was then further improved with a novel GFRBS design. The rule-based learning process in the GFRBS design was shifted to a combinatorial optimization problem and solved with a probabilistic and adaptive GA. Both of the two model outputs were enforced to satisfy production and attraction constraints in order to use them as part of sequential travel demand modelling. The performance of the models was evaluated comparatively with respect to the benchmark models: a traditional DCGM and a multilayer feed-forward NN. According to the results achieved, a straightforward consequence is that the FRBSs and the GFRBSs can be used to predict intra-city passenger flows with a high level of accuracy.

The present study also examined the proposed and benchmark models in many respects and achieved a variety of empirical results. In addition, all models were evaluated according to their simplicity, predictive ability, interpretability, flexibility, data dependency, etc. in trip distribution modelling. The main findings of the empirical analysis, shown in Table 5, can be summarized for the doubly constrained trip distribution case as follows: 

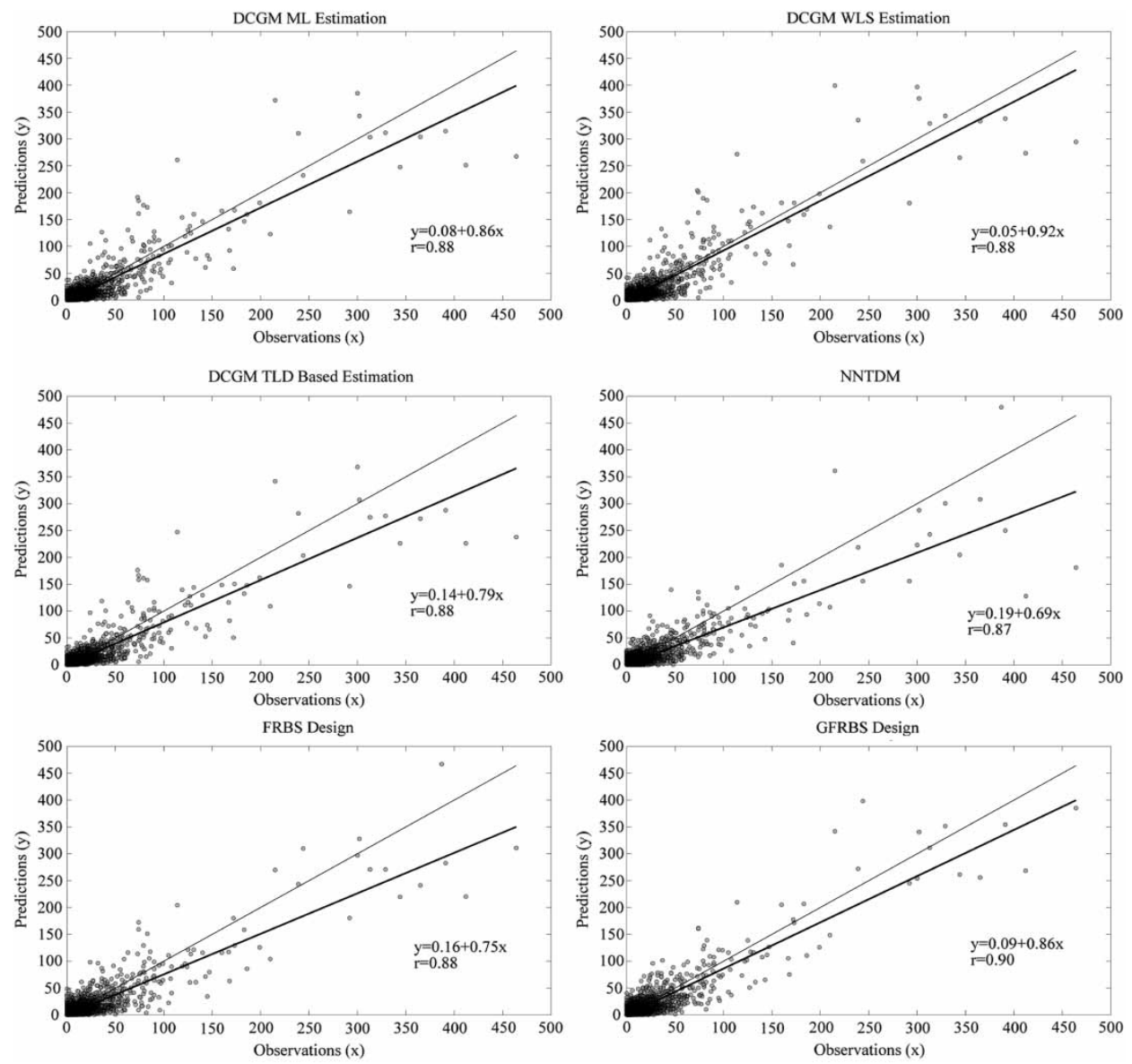

Figure 14. Regression plots - testing data set.

(1) traditional DCGMs are still simple and efficient; they are stable and robust, yet, they suffer from one-parameter generalization; they should be tried first in almost every case, at least for a comparison; they offer better predictions with ML or TLD-based parameter estimation.

(2) NNs may not show expected performance if they are forced to satisfy production-attraction constraints; they are unstable, non-interpretable and case/data dependent; their equation-free structure and potential usage with additional inputs provide an outstanding advantage.

(3) simply-designed FRBSs, learning from numerical data and expertise, are both interpretable and efficient in forecasting trip interchanges even if the data is large and noisy; they do not require data and can be established with only basic human reasoning; additional inputs can be introduced to the model easily as in NNs.

(4) GFRBSs offer a high level of accuracy in trip distribution modelling, although demand additional computation cost; they should be preferred especially when high accuracy is needed or when system complexity increases and classical rule based learning approaches fail. 
Table 4. Observed and modelled trip shares: intra-zonal vs. inter-zonal, intra-district vs. inter-district and bridge crossing vs. not bridge crossing trips.

\begin{tabular}{|c|c|c|c|c|c|c|c|}
\hline Trips & OBSERVED & $\begin{array}{c}\text { DCGM } \\
\text { ML } \\
\text { estimation } \\
(\%)\end{array}$ & $\begin{array}{c}\text { DCGM } \\
\text { WLS } \\
\text { estimation } \\
(\%)\end{array}$ & $\begin{array}{c}\text { DCGM } \\
\text { TLD } \\
\text { based } \\
\text { estimation } \\
(\%)\end{array}$ & $\begin{array}{c}\text { NNTDM } \\
(\%)\end{array}$ & $\begin{array}{c}\text { FRBS } \\
\text { design } \\
(\%)\end{array}$ & $\begin{array}{c}\text { GFRBS } \\
\text { design } \\
(\%)\end{array}$ \\
\hline $\begin{array}{l}\text { Intra- } \\
\text { Zonal }\end{array}$ & $24.5 \%$ & 24.3 & 27.0 & 21.4 & 18.6 & 18.5 & 21.7 \\
\hline $\begin{array}{l}\text { Percentage } \\
\text { Error }\end{array}$ & - & -1.1 & +9.8 & -12.9 & -24.1 & -24.4 & -11.8 \\
\hline $\begin{array}{l}\text { Inter- } \\
\text { Zonal }\end{array}$ & $75.5 \%$ & 75.7 & 73.0 & 78.6 & 81.4 & 81.5 & 78.3 \\
\hline $\begin{array}{l}\text { Percentage } \\
\text { Error }\end{array}$ & - & +0.3 & -3.20 & +4.2 & +7.8 & +7.9 & +3.8 \\
\hline $\begin{array}{l}\text { Intra- } \\
\text { District }\end{array}$ & $47.2 \%$ & 42.7 & 45.7 & 39.4 & 38.8 & 38.5 & 42.7 \\
\hline $\begin{array}{l}\text { Percentage } \\
\text { Error }\end{array}$ & - & -9.4 & -3.2 & -16.5 & -17.7 & -18.4 & -9.3 \\
\hline $\begin{array}{l}\text { Inter- } \\
\text { District }\end{array}$ & $52.8 \%$ & 57.3 & 54.3 & 60.6 & 61.2 & 61.5 & 57.3 \\
\hline $\begin{array}{l}\text { Percentage } \\
\text { Error }\end{array}$ & - & +8.4 & +2.8 & +14.7 & 15.8 & 16.4 & 8.3 \\
\hline $\begin{array}{l}\text { Bridge } \\
\quad \text { Crossing }\end{array}$ & $6.8 \%$ & 8.2 & 7.4 & 9.2 & 10.5 & 7.7 & 5.7 \\
\hline $\begin{array}{l}\text { Percentage } \\
\text { Error }\end{array}$ & - & +20.4 & +8.6 & +34.4 & +53.1 & +12.1 & -15.8 \\
\hline $\begin{array}{l}\text { Not } \\
\text { Bridge } \\
\text { Crossing }\end{array}$ & $93.2 \%$ & 91.8 & 92.6 & 90.8 & 89.5 & 92.3 & 94.3 \\
\hline $\begin{array}{l}\text { Percentage } \\
\text { Error }\end{array}$ & - & -1.5 & -0.6 & -2.5 & -3.8 & -0.9 & +1.2 \\
\hline
\end{tabular}

Note: The numbers given in bold show minimum scores for the percentage errors.

In conclusion, fuzzy and GFSs offer an alternative to traditional gravity models and NNs in modelling trip distributions. The present study has demonstrated their applicability to a challenging city region with a desired level of accuracy and interpretability. It differs from similar previous works (Kalic and Teodorovic, 1996, 2003 and Shafahi, Nourbakhsh, and Seyedabrishami, 2008) in several respects: for the first time a genetic fuzzy system has been proposed and applied to model intracity passenger flows; original solutions to the fuzzy rule base learning problem have been developed; and an extensive performance comparison has been established among the fuzzy, genetic fuzzy, DCGM and NN-based trip distribution model.

Further research could fruitfully explore the design of such fuzzy and GFSs with some new features, including: incorporating additional variables, for instance a zonal land use variable or a geographical barrier can be introduced as additional inputs; with the same variables but in different configurations such as with a Sugeno-type FRBS; with innovative evolutionary algorithms and learning strategies; and most 
Table 5. Model evaluation for the doubly-constrained trip distribution problem.

\begin{tabular}{|c|c|c|c|c|c|c|}
\hline Criteria & $\begin{array}{c}\text { DCGM } \\
\text { ML } \\
\text { estimation }\end{array}$ & $\begin{array}{c}\text { DCGM } \\
\text { WLS } \\
\text { estimation }\end{array}$ & $\begin{array}{l}\text { DCGM } \\
\text { TLD based } \\
\text { estimation }\end{array}$ & NNTDM & $\begin{array}{l}\text { FRBS } \\
\text { design }\end{array}$ & $\begin{array}{l}\text { GFRBS } \\
\text { design }\end{array}$ \\
\hline $\begin{array}{l}\text { Mathematical } \\
\text { simplicity }\end{array}$ & Moderate & Moderate & Strong & Moderate & Strong & Moderate \\
\hline $\begin{array}{l}\text { Application } \\
\text { simplicity }\end{array}$ & Strong & Strong & Strong & Moderate & Strong & Weak \\
\hline $\begin{array}{l}\text { Statistical } \\
\text { interpretability }\end{array}$ & Strong & Strong & Strong & Unknown & Unknown & Unknown \\
\hline $\begin{array}{l}\text { System } \\
\text { interpretability }\end{array}$ & Moderate & Moderate & Moderate & Weak & Strong & Strong \\
\hline Prediction ability & Moderate & Weak & Moderate & Weak & Moderate & Strong \\
\hline $\begin{array}{l}\text { Improvement } \\
\text { with additional } \\
\text { variables }\end{array}$ & Weak & Weak & Weak & Strong & Strong & Strong \\
\hline $\begin{array}{l}\text { Dependency to } \\
\text { data existence }\end{array}$ & Strong & Strong & Strong & Weak & Strong & Weak \\
\hline $\begin{array}{l}\text { Hybridization } \\
\text { ability }\end{array}$ & Weak & Weak & Weak & Strong & Strong & Strong \\
\hline $\begin{array}{l}\text { Computational } \\
\text { costs }\end{array}$ & Strong & Strong & Strong & Moderate & Strong & Weak \\
\hline $\begin{array}{l}\text { Ready to use } \\
\text { software } \\
\text { packages }\end{array}$ & Strong & Weak & Strong & Strong & Strong & Weak \\
\hline
\end{tabular}

importantly with an approximate FRBS design that works properly in a low quality data environment or under uncertainty and imprecision. Since field-collected data generally involves uncertainty, vagueness and incompleteness in transportation geography, FRBSs provide an outstanding opportunity to deal with these drawbacks.

\section{Acknowledgements}

The authors wish to thank the Greater Municipality of Istanbul for making the travel survey data available.

\section{References}

Avineri, E. 2005. "Soft Computing Applications in Traffic and Transport Systems: A Review." Advances in Soft Computing 1: 17-25. http://dx.doi.org/10.1007/3-540-32400-3_2.

Black, W. R. 1995. "Spatial Interaction Modeling Using Artificial Neural Networks." Journal of Transport Geography 3 (3): 159-166. doi:10.1016/0966-6923(95)00013-S.

Black, W. R. 2003. Transportation: A geographical analysis. New York: Guilford Press.

Bodenhofer, U., and F. Herrera. 1997. Ten Lectures on Genetic Fuzzy Systems, SCCH-TR0021. Hagenberg: Software Competence Center.

Celik, M. H. 2004. "Forecasting Interregional Commodity Flows Using Artificial Neural Networks: An Evaluation." Transportation Planning and Technology 27 (6): 449-467. doi:10.1080/0308106042000293499.

Chiou, Y. C., and L. W. Lan. 2005. "Genetic Fuzzy Logic Controller: An Iterative Evolution Algorithm with New Encoding Method." Fuzzy Sets and Systems 152 (3): 617-635. doi:10.1016/j.fss.2004.11.011. 
Cordon, O., F. Herrera, F. Hoffmann, and L. Magdalena. 2001. Genetic Fuzzy Systems: Evolutionary Tuning and Learning of Fuzzy Knowledge Bases. Singapore: World Scientific.

Cordón, O., F. Gomide, F. Herrera, F. Hoffmann, and L. Magdalena. 2004. "10 years of Genetic Fuzzy Systems: Current Framework and New Trends.” Fuzzy Sets and Systems 141 (1): 5-31. doi:10.1016/S0165-0114(03)00111-8.

Demuth, H., M. Beale, and M. Hagan. 2009. Neural Network Toolbox 6: Users Guide. Natick, MA: The MathWorks, Inc.

Dickey, J. W. 1983. Metropolitan Transportation Planning. 2nd ed. New York: McGraw-Hill.

Dougherty, M. 1995. "A Review of Neural Networks Applied to Transport." Transportation Research Part C: Emerging Technologies 3 (4): 247-260. doi:10.1016/0968-090X(95)00009-8.

Easa, S. M. 1993. "Urban Trip Distribution in Practice 1: Conventional Analysis." Journal of Transportation Engineering 119 (6): 793-815. doi:10.1061/(ASCE)0733-947X(1993)119:6(793).

Evans, A. W. 1971. "The Calibration of Trip Distribution Models with Exponential or Similar Cost Functions." Transportation Research 5 (1): 15-38. doi:10.1016/0041-1647(71)90004-9.

Fischer, M. M., and S. Gopal. 1994. "Artificial Neural Networks - A New Approach to Modeling Interregional Telecommunication Flows." Journal of Regional Science 34 (4): 503-527. doi:10.1111/j.1467-9787.1994.tb00880.x.

Fotheringham, A. S., and D. C. Knudsen. 1987. Goodness-of-Fit Statistics. Norwich: Geo Books.

Fotheringham, A. S., and M. E. O'Kelly. 1989. Spatial Interaction Models: Formulations and Applications. Dordrecht: Kluwer Academic Publishers.

Furness, K. P. 1965. "Time Function Iteration." Traffic Engineering \& Control 7: 458-460.

Goldberg, D. E. 1989. Genetic Algorithms in Search, Optimization, and Machine Learning. Boston: Addison-Wesley.

Haykin, S. 1999. Neural Networks: A Comprehensive Foundation. 2nd ed. India: Pearson Prentice Hall.

Herrera, F. 2008. "Genetic Fuzzy Systems: Taxonomy, Current Research Trends and Prospects.” Evolutionary Intelligence 1 (1): 27-46. doi:10.1007/s12065-007-0001-5.

Holland, J. H. 1975. Adaptation in Natural and Artificial Systems. Ann Arbor: University of Michigan Press.

Hornik, K., M. Stinchcombe, and H. White. 1989. "Multilayer Feedforward Networks are Universal Approximators." Neural Networks 2 (5): 359-366. doi:10.1016/0893-6080(89) 90020-8.

Hornik, K. 1991. "Approximation Capabilities of Multilayer Feedforward Networks." Neural Networks 4 (2): 251-257. doi:10.1016/0893-6080(91)90009-T.

Hyman, G. M. 1969. "The Calibration of Trip Distribution Models." Environment and Planning A 1 (1): 105-112. doi:10.1068/a010105.

Ishibuchi, H. 2007. "Multiobjective Genetic Fuzzy Systems: Review and Future Research Directions." In Fuzzy Systems Conference, FUZZ-IEEE 2007. IEEE International, 1-6. Imperial College, London, UK, July 23-26.

Istanbul Metropolitan Municipality Transportation Planning Department. 2008. Istanbul Transportation Master Plan Household Survey: Analytic Study and Model Calibration. Istanbul: Istanbul Metropolitan Municipality.

Kalić, M., and D. Teodorovic. 2003. "Trip Distribution Modelling Using Fuzzy Logic and A Genetic Algorithm.” Transportation Planning and Technology 26 (3): 213-238. doi:10.1080/ 0308106032000154575.

Kalic, M. and D. Teodorovic. 1996. "Solving the Trip Distribution Problem by Fuzzy Rules Generated by Learning from Examples (in Serbian)." In Proceedings of the XXIII Yugoslav Symposium on Operations Research, 777-780. Zlatibor, Yugoslavia, October 1-5.

Kanafani, A. 1983. Transportation Demand Analysis. New York: McGraw-Hill.

Knudsen, D. C., and A. S. Fotheringham. 1986. "Matrix Comparison, Goodness-of-Fit and Spatial Interaction Modeling." International Regional Science Review 10 (2): 127-147. doi:10.1177/016001768601000203.

Kosko, B. 1994. "Fuzzy Systems as Universal Approximators." IEEE Transactions on Computers 43 (11): 1329-1333. doi:10.1109/12.324566.

Mamdani, E. H. 1974. "Application of Fuzzy Algorithms for Control of Simple Dynamic Plant." Proceedings of IEEE 121 (12): 1585-1588. http://dx.doi.org/10.1049/piee.1974.0328. 
Mamdani, E. H., and S. Assilian. 1975. "An Experiment in Linguistic Synthesis with A Fuzzy Logic Controller." International Journal of Man-Machine Studies 7 (1): 1-13. doi:10.1016/ S0020-7373(75)80002-2.

Mozolin, M., J.-C. Thill, and E. Lynn Usery. 2000. “Trip Distribution Forecasting with Multilayer Perceptron Neural Networks: A Critical Evaluation." Transportation Research Part B: Methodological 34 (1): 53-73. doi:10.1016/S0191-2615(99)00014-4.

Munakata, T. 2008. Fundamentals of The New Artificial Intelligence: Neural, Evolutionary, Fuzzy and More. 2nd ed. London: Springer-Verlag.

Mussone, L. 1999. "A Review of Feedforward Neural Networks in Transportation Research." e \& i Elektrotechnik und Informationstechnik 116 (6): 360-365.

Openshaw, S. 1993. "Modeling Spatial Interaction Using Neural Net." In Geographical Information Systems, Spatial Modeling, and Policy Evaluation, edited by M. M. Fischer and P. Nijkamp, 147-164. New York: Springer-Verlag.

Oppeneim, N. 1995. Urban Travel Demand Modeling: From Individual Choices to General Equilibrium. New York: Wiley Interscience.

Ortuzar, J. D., and L. G. Willumsen. 2001. Modelling Transport. 3rd ed. Chichester: John Wiley \& Sons.

Pedrycz, J. 1996. Fuzzy Sets Engineering. Boca Raton: CRC Press.

Ross, T. J. 2004. Fuzzy Logic with Engineering Applications. 2nd ed. New York: John Wiley \& Sons.

Roy, J. R. 2004. Spatial Interaction Modelling: A Regional Science Context. Berlin: SpringerVerlag.

Sadek, A. W. 2007. "Artificial Intelligence Applications in Transportation." Transportation Research Circular E-C113: Artificial Intelligence in Transportation Information for Application. Washington, DC: Transportation Research Board.

Sen, A., and S. Soot. 1981. "Selected Procedures for Calibrating the Generalized Gravity Model." Papers of the Regional Science Association 48 (1): 165-176. doi:10.1007/ BF01940997.

Sen, A. 1986. "Maximum-Likelihood-Estimation of Gravity Model Parameters." Journal of Regional Science 26 (3): 461-474. doi:10.1111/j.1467-9787.1986.tb01054.x.

Sen, A., and T. E. Smith. 1995. Gravity Models of Spatial Interaction Behavior. Berlin: Springer-Verlag.

Shafahi, Y., S. M. Nourbakhsh, and S. Seyedabrishami. 2008. "Fuzzy Trip Distribution Models for Discretionary Trips." In 11th International IEEE Conference on Intelligent Transportation Systems, ITSC 2008: 557-562.

Smith, D. P., and B.G. Hutchinson. 1981. "Goodness of Fit Statistics for Trip Distribution Models." Transportation Research Part A: General 15 (4): 295-303. doi:10.1016/01912607(81)90011-X.

Smith, S. F. 1980. "A Learning System Based on Genetic Adaptive Algorithms." PhD thesis., University of Pittsburgh.

Sugeno, M., and G. T. Kang. 1988. "Structure Identification of Fuzzy Model." Fuzzy Sets and Systems 28 (1): 15-33. doi:10.1016/0165-0114(88)90113-3.

Takagi, T., and M. Sugeno. 1985. "Fuzzy Identification of Systems and Its Applications to Modeling and Control." IEEE Transactions on Systems, Man, and Cybernetics 15 (1): 116-132. doi:10.1109/TSMC.1985.6313399.

Tapkın, S., and Ö. Akyılmaz. 2009. "A New Approach to Neural Trip Distribution Models: NETDIM." Transportation Planning and Technology 32 (1): 93-114. doi:10.1080/ 03081060902750710.

Teodorović, D. 1994. "Fuzzy Sets Theory Applications in Traffic and Transportation." European Journal of Operational Research 74 (3): 379-390. doi:10.1016/0377-2217(94)902186.

Teodorović, D., and K. Vukadinovic. 1998. Traffic Control and Transport Planning: A Fuzzy Sets and Neural Networks Approach. Dordrecht: Kluwer.

Teodorović, D. 1999. "Fuzzy Logic Systems for Transportation Engineering: The State of The Art." Transportation Research Part A: Policy and Practice 33 (5): 337-364. doi:10.1016/ S0965-8564(98)00024-X. 
Tillema, F., K. M. van Zuilekom, and M. F. A. M. van Maarseveen. 2006. "Comparison of Neural Networks and Gravity Models in Trip Distribution." Computer-Aided Civil and Infrastructure Engineering 21: 104-119. doi:10.1111/j.1467-8667.2005.00421.x.

Transportation Research Board. 1998. Travel Estimation Techniques for Urban Planning: NCHRP Report 365. Washington, DC: National Academy Press.

Transportation Research Board. 2007. Transportation Research Circular E-C113: Artificial Intelligence in Transportation Information for Application. Washington, DC: Transportation Research Board of the National Academies.

TurkStat. 2010. Press Release: Address Based Population Registration System Population Census Results, 2009. Ankara: Turkish Statistical Institute, No: 15.

Wang, L. X., and J. M. Mendel. 1992. "Generating Fuzzy Rules by Learning from Examples." IEEE Transactions on Systems, Man, and Cybernetics 22 (6): 1414-1427. doi:10.1109/ 21.199466.

Wilson, A. G. 1967. "A Statistical Theory of Spatial Distributions." Transportation Research 1: 253-269. doi:10.1016/0041-1647(67)90035-4.

Wilson, A. G. 1970. Entropy in Urban and Regional Modelling. London: Pion.

Zadeh, L. A. 1965. "Fuzzy Sets." Information and Control 8 (3): 338-353. doi:10.1016/S00199958(65)90241-X.

Zadeh, L. A. 1973. "Outline of a New Approach to the Analysis of Complex Systems and Decision Processes." Systems, Man and Cybernetics, IEEE Transactions on, SMC-3 (1): 28-44. http://dx.doi.org/10.1109/TSMC.1973.5408575. 